University of Louisville

ThinkIR: The University of Louisville's Institutional Repository

Electronic Theses and Dissertations

$5-2010$

\title{
Comparison of clinically projected and radiographically optimal implant fixture placement.
}

William Shane Vaughn 1984-
University of Louisville

Follow this and additional works at: https://ir.library.louisville.edu/etd

\section{Recommended Citation}

Vaughn, William Shane 1984-, "Comparison of clinically projected and radiographically optimal implant fixture placement." (2010). Electronic Theses and Dissertations. Paper 1487.

https://doi.org/10.18297/etd/1487

This Master's Thesis is brought to you for free and open access by ThinkIR: The University of Louisville's Institutional Repository. It has been accepted for inclusion in Electronic Theses and Dissertations by an authorized administrator of ThinkIR: The University of Louisville's Institutional Repository. This title appears here courtesy of the author, who has retained all other copyrights. For more information, please contact thinkir@louisville.edu. 


\title{
COMPARISON OF CLINICALLY PROJECTED AND RADIOGRAPHICALLY OPTIMAL IMPLANT FIXTURE PLACEMENT
}

\author{
By \\ William Shane Vaughn \\ B.S., Kentucky Wesleyan College, 2006
}

\begin{abstract}
A Thesis
Submitted to the Faculty of the Graduate School of the University of Louisville In Partial Fulfillment of the Requirements

For the Degree of
\end{abstract}

Master of Oral Biology

University of Louisville

Louisville, Kentucky

May 2010 


\title{
Comparison of Clinically Projected and Radiographically Optimal Implant Fixture Placement
}

\author{
By \\ William Shane Vaughn \\ B.S., Kentucky Wesleyan College, 2006
}

A Thesis Approved on

$$
\text { March } 17^{\text {th }} 2010
$$

By the following Thesis Committee:

Dr. Allan G. Farman, Thesis Director

$\overline{\text { Dr. William C. Scarfe, Thesis Co-Director }}$

$\overline{\text { Dr. Bryan T. Harris, Committe Member }}$

$\overline{\text { Dr. Mary M. Paris, Committe Member }}$ 


\section{ACKNOWLEDGEMENTS}

I would like to recognize and thank the following individuals:

Dr. Allan G Farman, Thesis Director, for giving me the opportunity to conduct this research, for his excitement in the study, and for his insightful guidance and encouragement throughout the project.

Dr. William C Scarfe, Thesis Co-Director, also for giving me the opportunity to conduct this research, for his many hours of help and support of this endeavor, and for his interest in this project.

Dr. Bryan T Harris, Thesis Committee Member, for his helpful insight regarding the planning and placement of dental implants from the perspective of a prosthedontist as well as his support and guidance throughout the project.

Dr. Mary M Paris, Thesis Committee Member, for her insight into implant planning and placement from the perspective of a general practitioner as well as her support and encouragement.

My labmates Elizabeth Boyle and Allison Lohse, who are genuinely amazing people, for making this a truly enjoyable experience. 


\title{
ABSTRACT \\ COMPARISON OF CLINICALLY PROJECTED AND RADIOGRAPHICALLY OPTIMAL IMPLANT FIXTURE PLACEMENT
}

\author{
William Shane Vaughn
}

March 31, 2010

Potential dental implant site assessment is initially determined clinically. Subsequent radiographic assessment also provides information about the quantity of available bone. It was hypothesized that the use of radiographic markers in mandibular stents to project the clinically derived placement of implant fixtures would not correspond to the optimal trajectory of the fixture based on acceptable implant placement criteria applied on Cone Beam Computed Tomography images. A retrospective audit of patients with edentulous mandibles scanned with a surgical guide was completed. A total of 218 potential implant sites in 77 patients were evaluated utilizing an implant planning software package (EZ Guide V1.3; Aspen Dental, Burlington, MA). Projections were found to deviate from optimal placement in more than $93 \%$ of sites. In more than $75 \%$ of such sites, the clinically projected position was buccal to optimal placement. The results suggest that clinical assessment alone is inadequate to project optimal implant fixture position.

KEY WORDS: dental implant $\bullet$ CBCT $\bullet$ cone beam $\bullet$ radiographic marker $\bullet$ projection 
TABLE OF CONTENTS

PAGE

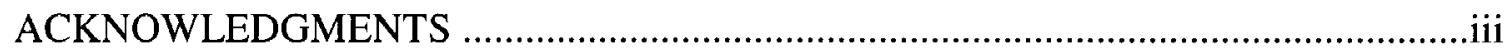

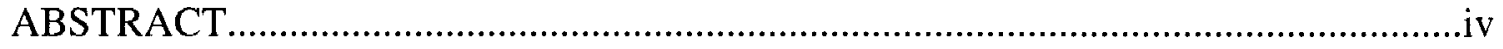

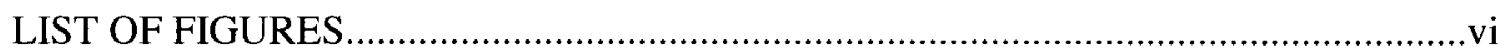

\section{CHAPTER}

I. INTRODUCTION .................................................

Background and Significance.......................................................

Objectives.........................................................

Specific Aims..................................................... 3

II. REVIEW OF THE LITERATURE................................4

The Role of Radiographic Imaging in Implant Site

Assessment.........................................................

History of Dental Implants.........................................

Guidelines for Dental Implant Fixture

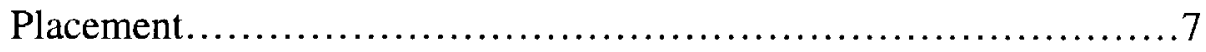

Types of Surgical Guides.................................... 10

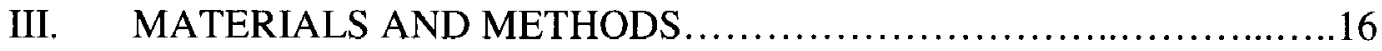

Hypothesis......................................................... 16

Research Design................................................. 16

Qualitative Assessment.......................................24 
Quantitative Assessment.

Statistical Analysis............................................28

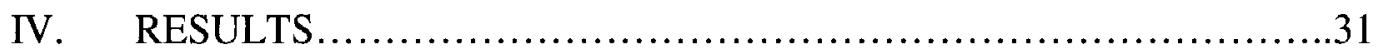

Surgical Guide Status....................................... 31

Subject Sample Demographics.........................................33

Analysis of Projected Fixture Placement........................................33

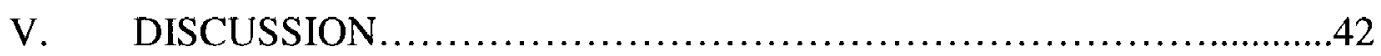

Purpose and Motivation..........................................42

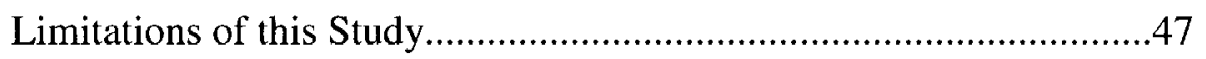

Future Areas of Research.........................................48

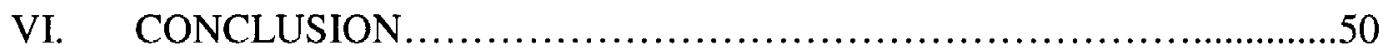

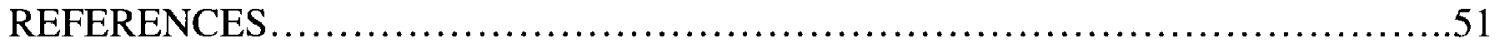

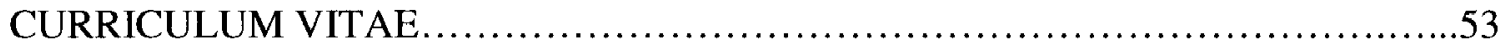




\section{LIST OF FIGURES}

FIGURE

PAGE

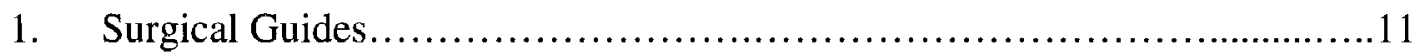

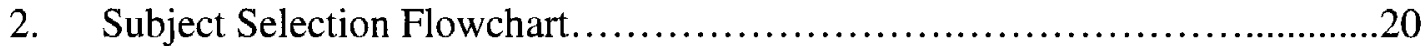

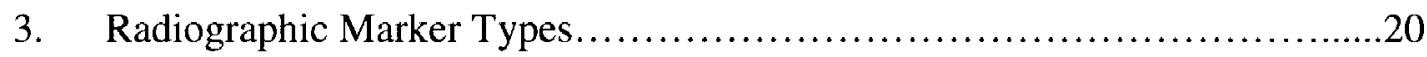

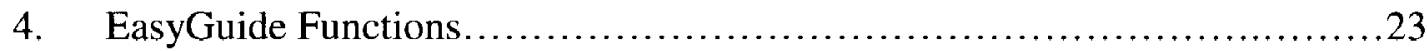

5. Implant Fixture Projections within Alveolar Bone (Lateral Position............24

6. Projected Implant Fixture Complications................................24

7. Optimal and Projected Implant Fixtures...................................25

8. Angular Deviation Calculation in EasyGuide.............................26

9. Restorative Space and Residual Bone Trajectory Measurements.............27

10. Frequency Distribution: Surgical Guides by Number of Radiographic

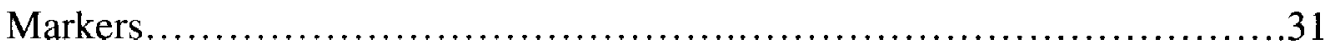

11. Frequency Distribution: Radiographic Markers by Projected Location...........32

12. Frequency Distribution: Radiographic Markers by Projected Placement.......33

13. Frequency Distribution: Acceptable/Unacceptable Projections ........................34

14. Projected Trajectory Based on Displacement from Optimal....................35

15. Projected Trajectory Based on Displacement from Optimal (Acceptable only)

16. Frequency Distribution: Potential Complications by Site....................37

17. Frequency Distribution: Modifications by Site.............................38 
18. Quantitative analysis: Projected vs. Optimal.............................40 


\section{CHAPTER I}

\section{INTRODUCTION}

\section{Background and Significance}

For many years two-dimensional (2D) plain film intra- and extra-oral radiography has been used for the assessment of potential alveolar sites in the maxilla and mandible for dental implant placement. Cross-sectional imaging, such as tomography or, more recently, computed tomography has also been used, however access to and technical expertise in the use of these techniques has been somewhat limited. The availability of cone beam computed tomography (CBCT) now provides a low dose digital alternate producing images in both 2- and 3-D providing accurate visualization and facilitating implant treatment planning.

Long-term success of an endosseous dental implant has significant correlation to surgical technique, the three-dimensional position in bone, biomechanics, and biologic response. Advances in implant design, and surface technology have also affected success. Dental implants are surgically placed, many times facilitated by a surgical guide or template to a restoratively predetermined position. A surgical guide is " a guide, derived from the diagnostic wax-up, used to assist in the preparation for and placement of dental implants. It dictates drilling position and angulation." ${ }^{6}$ A number of types of surgical guides and templates have been described in the literature. ${ }^{7-10}$ Ideally a surgical 
guide should be constructed based on a pre-surgical diagnostic workup with radiographic markers to direct implant placement in three dimensions, (1) bucco-lingually, (2) mesiodistally, and (3) apicocoronally. ${ }^{11}$ The American Academy of Oral and Maxillofacial Radiology recommends the use of surgical guides with "radiopaque indicators...preferably based on diagnostic wax-ups...(to) verify implant sites" ${ }^{12}$

The Faculty Private Practice of Dr. Allan Farman and Dr. William Scarfe, located in the Department of Surgical and Hospital Dentistry at the University of Louisville School of Dentistry, has worked with Cone Beam Computed Tomography for more than five years and has accumulated a database of over 2,000 clinical image volumes. Included in these datasets are numerous patients who have been scanned with radiographic surgical guides depicting the clinically projected implant trajectory of dental implant fixtures.

\section{Objectives}

The main purpose of this study is to compare and correlate the positions of projected implant fixture placement using cylindrical radiopaque markers in mandibular edentulous regions with optimal placement guidelines facilitated by 3D virtual implant placement. The subject sample will be derived from a retrospective audit of the radiologic database of patients referred to oral and maxillofacial faculty practice. The understanding of this correlation should aid in the development of selection criteria for use of radiographic surgical guides and $\mathrm{CBCT}$ in dental implant planning. Despite the frequency of dental implant placement no such guidelines for CBCT image selection currently exist. ${ }^{5}$ 


\section{Specific Aims}

1. Describe the demographics of patients who are referred for CBCT imaging for the purpose of planning a mandibular, implant-retained overdenture.

2. Describe the location, number, and projected status of the implant fixture projections obtained from clinically derived cylindrical radiographic markers on surgical guides.

3. Describe the availability of the residual alveolar bone with respect to the placement of an implant fixture.

4. Describe unsuitable projections with respect to the remaining projections in surgical guides.

5. Describe potential complications with implant fixtures placed based on the trajectory of the clinically derived radiographic markers.

6. Describe and quantify the deviation of projected implant fixtures from radiographically optimal placement. 


\section{CHAPTER II}

\section{REVIEW OF THE LITERATURE}

\section{The Role of Radiographic Imaging in Implant Site Assessment}

The radiologic evaluation of a patient for the placement of dental implants has been performed by utilizing intraoral, cephalometric, panoramic radiographs, tomography, or a combination of any or all of these methods. These images allow the restorative clinician to develop a plan of treatment for placing the dental implants based on the patient's bony anatomical structure. In more recent years, both conventional and computed tomography have been utilized more frequently for assessing potential dental implant sites because of the ability to construct buccolingual cross-sectional images. ${ }^{12}$

The American Academy of Oral and Maxillofacial Radiology (AAOMR) has outlined five basic considerations for the radiographic imaging of potential dental implant fixture sites. These considerations include:

1) There should be an adequate imaging in order to provide needed anatomic information such as quantity and quality of bone and location of anatomic structures.

2) The type of imaging utilized should provide the required information with both adequate precision and accuracy for implant planning. 
3) There should be a method of relating images to the individual patient's anatomy. This usually involves the utilization of a radiographic template with radiopaque markers.

4) Whatever imaging technique is used, effort should be made to reduce image distortion as much as possible.

5) Consideration for imaging dosage and cost to the patient should be made. If more than one modality is indicated, the technique with the lowest reasonable dose of radiation should be utilized. ${ }^{12}$

CBCT is important in the preoperative evaluation of the morphology of the jaw and extent of alveolar ridge resorption to determine the feasibility of placing dental implants at specific sites. In addition it ensures that every possible precaution has been made to reduce the risk of unintended involvement of vital structures such as the neurovascular bundle within the inferior alveolar canal in the lower jaw, and the sinuses and nasal fossa in the upper jaw. CBCT can also be used to evaluate the location of previously placed implants.

Shindoda, et al. (2004) reported on the frequency and referral pattern distribution of CBCT utilization for the assessment of potential dental implant sites on 3,000 cases over a one year period at their institution. Their referral patterns are reflective of their available equipment which provides a limited area CBCT (3D Accustomed). They reported that approximately $50 \%$ of referrals were for implant site assessment with $20 \%$ for temporomandibular disease (TMD). The remainder of cases included apical disease, impactions, supernumeraries and pathology. 
The AAOMR recognizes $\mathrm{CBCT}$ as a major advancement in the imaging armamentarium available to the dental profession. The AAOMR is currently constructing a position paper regarding the appropriate application of CBCT to provide evidencebased guidelines. Currently, the AAOMR has formulated a set of guidelines to assist practitioners in providing appropriate CBCT radiologic care. Among these guidelines is stated the need for documentary evidence which demonstrates the diagnostic or treatment guidance need for the CBCT examination. ${ }^{5}$

\section{History of Dental Implants}

Essentially four basic types of dental implants have been marketed for the purpose of restoring oral form and function. These include subperiosteal implants, blade implants, transosseous implants, and endosseous implants.

Subperiosteal implants are still sometimes utilized in cases with advanced bone resorption. This implant involves placing a supportive structure for the restoration on top of the bone but under the mucosa. Blade implants are still sometimes placed in some cases in which the residual alveolar bone lacks bucco-lingual width. This procedure involves creating a thin slice in the alveolar bone a placing a thin sheet-like metal fixture in the crevice for the support of the restoration. Transosseous implants are similar to endosseous implant in that they are placed within bone; however, they protrude through the other side and are secured on the other side, similar to a screw and nut. Transosseous implants are rarely utilized today as they require greater cost and surgery/recovery time with little to no added benefit. Root form endosseous dental implants are normally placed within the residual alveolar bone but, unlike transosseous implants, do not 
protrude through the other side. They are normally made of titanium and threaded and/or coated in order to provide greater surface area for osseointegration of the fixture. Today, based on scientific data and long-term success rates, endosseous implants are the most preferred for restoration retention, when placement is possible.

Endosseous dental implants were introduced following the discovery of osseointegration by Brånemark in 1952. Brånemark discovered osseointegration while studying the healing process of microscopic bony defects in rabbits. He found that microscope heads, which were made out of titanium and had been placed in the bony tissue of the rabbits to study the healing processes, could not be easily removed at the end of the study due to the integration of the titanium metal into the bone. This concept was expanded into the area of dental restoration and was used create implant fixtures that could be integrated into the alveolar bone in order to support fixed prostheses. ${ }^{18}$

Since the discovery of osseointegration by Brånemark numerous implant fixture diameters, designs, platforms, abutments, lengths and surfaces have been developed and marketed by manufacturers. Binon (2000) outlined more than 1,300 dental implant bodies that have been marketed by various manufacturers. In general, however, these implant fixtures are all composed of titanium with some sort of surface treatment or design to create greater surface area to encourage osseointegration. ${ }^{19}$

\section{Guidelines for Dental Implant Fixture Placement}

\section{Physical Considerations}

The following information should be obtained from the pre-operative imaging: 
1) Presence of disease which should be addressed before continuing with planned implant procedures.

2) The location of anatomic features that should be avoided when placing the implant fixture (e.g. mandibular canal, mental foramen, maxillary sinus, and nasopalatine canal).

3) The location of osseous morphology (e.g. knife-edge ridge, developmental variations, and cortical integrity).

4) The amount of bone available for the placement of the implant fixture. ${ }^{12}$

It is well documented in the literature that it is preferable, for the long term success of endosseous dental implant fixtures, that there be $1-1.5 \mathrm{~mm}$ of alveolar bone on either side (bucco-lingual) of the fixture after placement. It is also preferable to have at least $1-2 \mathrm{~mm}$ of bone separating the dental implant fixture from any adjacent vital structures (e.g. mandibular canal, maxillary sinus, surrounding tooth root structures). ${ }^{13}$ Specifically, it is preferable to have at least $2 \mathrm{~mm}$ separating the apex of the implant fixture from the coronal aspect of the mandibular nerve and $1.5-2 \mathrm{~mm}$ separating the implant fixture from any adjacent tooth root. ${ }^{15}$

Five millimeters of alveolar bone mesial to the mental foramen has also been recommended as a good general guideline regarding the placement of implant fixtures. This is due to complications that can occur in subjects with an anterior loop of the mandibular canal. However, due to variation between individual subjects, no guideline can be considered completely safe. Therefore it is advisable to analyze each individual subject on a case by case basis. It has also been noted that identification and linear 
measurements of the mandibular canal are much more accurate when imaged with CBCT than with conventional two-dimensional radiographs. ${ }^{15}$

Non compatible trajectory between the residual bone and planned implant trajectory can be compensated for by using alternative courses of treatment, such as angled abutments or bone grafting, or by changing treatment plans altogether. Moderate differences of $2^{0}-20^{0}$ may be compensated for with the use of angled abutments. Extreme deviation $\left(>20^{\circ}\right)$ between the residual bone and the prosthetic trajectories may result in biomechanical, technical, and esthetic concessions. Another important aspect is the horizontal relationship of the projected implant fixture trajectory (PPT). Even though there might be some angle deviation from the trajectory of the residual alveolar ridge (RBT), the implant might be directed toward the center of the bulk of residual bone. Likewise, an implant trajectory may be consistent with the RBT, but be positioned horizontally either buccal or lingual to it. Either situation will necessitate a change in the treatment plan (a moderate change in the direction of the surgical indicator or a bone grafting procedure)..$^{8}$

\section{Number of Fixtures}

It has been well documented that two implant fixtures in a mandibular implant retained overdenture is adequate for restoration retention. Previously, four implant fixtures had been indicated; however, more recent literature has shown that the placement of four (or more) implant fixtures has no benefit to the edentulous patient with regard to the success of the fixtures or the retention of the restoration. Placement of more than two fixtures for the purpose of restoration retention is only recommended for scenarios in 
which the maxilla is dentate, implant fixtures are $<8 \mathrm{~m}$ in length and $<3.5 \mathrm{~mm}$ in diameter, patients with specific anatomical considerations, or patients with high retention needs. ${ }^{17}$

\section{Crown-Height Space}

The crown-height space requirement for an implant retained overdenture is 14$10.5 \mathrm{~mm}$, with less than $10.5 \mathrm{~mm}$ being the space at which the restoration becomes compromised. At this level the strength of the restoration is not only compromised, but the space available for the abutment is compromised, resulting in decreased retention. The space available for esthetic considerations is also reduced and poor hygiene conditions compromise long-term maintenance. ${ }^{16}$ In some cases in which the restoration would be compromised by lack of crown-height space, space can be created by surgically reducing the residual alveolar bone (alveoloplasty) in order to create an appropriate amount of space for the restoration.

\section{Types of Surgical Guides}

CBCT imaging can be utilized for the planning of dental implants. This data can then be utilized for the planning of the location of the implant fixture by creating a non-restrictive guide which is created by the planning clinician for the implant surgeon. Implant planning can be completed electronically using various software programs which can be utilized to create a restrictive guide through stereolithography by sending the treatment plan out to a third-party company to complete the construction of the guide. Both tissue and bone-based restrictive guides are examples of surgical guides manufactured through stereolithography. 


\section{Tissue-Based Restrictive Guides}

These guides are generally utilized for flapless surgeries in which a hole is punched through the tissue and access to the alveolar bone is obtained without laying a flap in order to place the implant fixture. These guides are very restrictive to the surgeon placing the implant fixture and must, therefore, be very accurate in their projected placement location and trajectory. These are normally very expensive due to the planning involved to ensure proper planning and fabrication.

\section{Bone Based Restrictive Guides}

Bone based restrictive guides are also very restrictive to the surgeon, however, rather than fitting over the soft tissue of the oral cavity, these are fitted to the bone once the soft tissue has been opened and pulled away from the bone. Like tissue-based guides, bone based guides must also be very accurate in their projected position and trajectory as they leave no room for modifications by the surgeon. Bone-based guides are also very expensive as the planning and fabrication must be done very carefully to ensure accuracy. Restrictive guides are more commonly used when very precise placement is needed, such as large fixed implant reconstructions.

\section{Non-Restrictive Guides}

Non-restrictive guides provide the surgeon with a template for the projected position and trajectory of the implant fixture but leave some room to manipulate drill position while in surgery. These are generally much less expensive than restrictive 
guides and can usually be fabricated by the referring clinician based on the diagnostic wax up and surgical guide used for imaging.

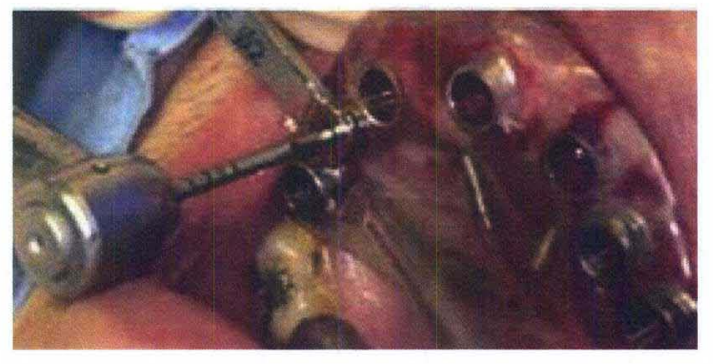

Tissue-Based Restrictive Guide

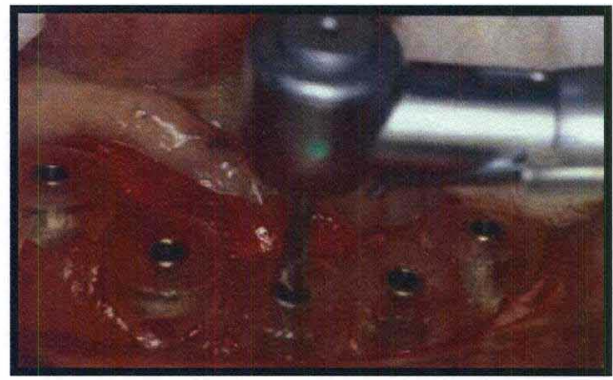

Bone-Based Restrictive Guide

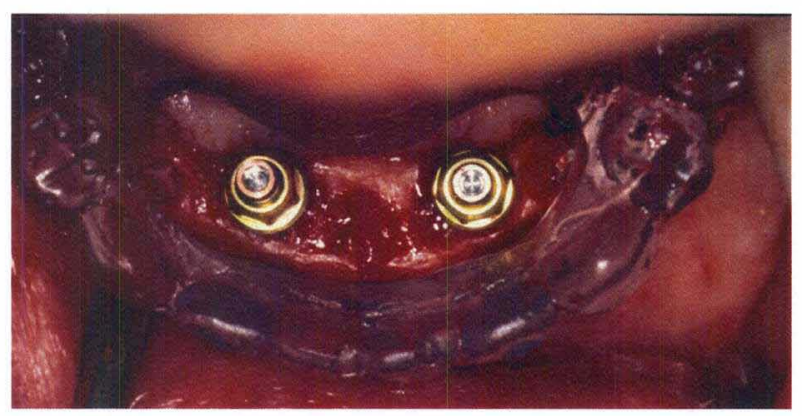

Non-Restrictive Guide

Figure B1: Examples of Restrictive and non-restrictive guides.

\section{Current Dental Implant Topics}

Previously researchers have investigated variability between projected and actual implant placement using restrictive surgical guides. We have found no studies in the literature comparing clinically derived, prosthetically-driven potential implant projected position with optimal theoretical placement based on available alveolar bone.

Almog and Sanchez analyzed the amount of deviation between planned prosthetic trajectory on using surgical guides and residual bone trajectory on cross-sectional 
tomographic slices at 235 implant sites of the maxillary and mandibular dental arches on 92 patients. They found the greatest discrepancy between the planned prosthetic trajectory (PPT) and the residual bone trajectories (RBT) in the mandibular molar area $\left(24.3^{0} \pm 12^{0}\right) .^{7}$

Ersoy, et al. compared the locations and axes of virtual 3D placement of 94 implants derived from surgical guides on CT scans to post operative placement using restrictive surgical guides. Compared to the planned implants, the placed implants showed angular deviation of $4.9^{\circ}-2.36^{\circ}$, whereas the mean linear deviation was $1.22-$ $0.85 \mathrm{~mm}$ at the implant neck and $1.51-1 \mathrm{~mm}$ at the implant apex. Compared to the implant planning, the angular deviation and linear deviation at the neck and apex of the placed maxillary implants were $5.31^{\circ}-0.36^{\circ}, 1.04-0.56 \mathrm{~mm}$, and $1.57-0.97 \mathrm{~mm}$, respectively, whereas corresponding figures for placed mandibular implants were $4.44^{\circ}-$ $0.31^{\circ}, 1.42-1.05 \mathrm{~mm}$, and $1.44-1.03 \mathrm{~mm}$, respectively. These deviations most likely reflect differences due to surgical accuracy rather than inaccuracies in $\mathrm{CBCT}$ measurements and planning.

Horwitz, et al. compared control surgical guides (C-group) with software planned guides (E-group) in resin models to determine the accuracy of CBCT guided implant planning. Their results revealed an mean apex depth deviations for $\mathrm{E}$ and $\mathrm{C}$ implants $[0.49 \mathrm{~mm} \pm 0.36$ standard deviation (SD) and $0.32 \mathrm{~mm} \pm 0.21 \mathrm{SD}$, respectively], and the mean apex radial deviations $(0.63 \mathrm{~mm} \pm 0.38 \mathrm{SD}$ and $0.49 \mathrm{~mm} \pm 0.17 \mathrm{SD}$, respectively) were similar (P40.05). The mean angulation deviations for $\mathrm{E}$ and $\mathrm{C}$ implants were $2.17 \pm$ $1.061 \mathrm{SD}$ and $1.33 \pm 0.691 \mathrm{SD}, \mathrm{P}<0.05$. The surgical guides were also grouped into groups A (performed with unused drill guides) and group B (performed with previously 
used drill guides). The greater deviations in group B over group A also suggest a correlation between greater deviations and multiple usage of the surgical guides. ${ }^{3}$

Nickenig, et al. showed similar results with CBCT image derived restrictive surgical guides from 102 patients (total of 250 implants) showing high predictability of implant fixture size (only one had to be reduced in diameter), low percentage of unusable guides (delayed implant placement was necessary in eight cases), and high percentage (58.1\%) of completely flapless surgery being realized through CBCT planning. ${ }^{2}$

Van Assche, et al. analyzed the accuracy of dental implant planning in four partially edentulous cadaver mandibles. By using CBCT data to create stereolithographic drill guides, they showed an average deviation of $2^{\circ}$ (SD: 0.8 , range: $0.7-4.01$ ) in the placed implant fixtures, as compared with the planning, while the mean linear deviation was $1.1 \mathrm{~mm}$ (SD: $0.7 \mathrm{~mm}$, range $0.3-2.3 \mathrm{~mm}$ ) at the hex and $2.0 \mathrm{~mm}$ (SD: $0.7 \mathrm{~mm}$, range $0.7-2.4 \mathrm{~mm}$ ) at the tip. These deviations could be due in part to the support of the surgical guide by both hard (teeth) and soft (mucosa) tissues in the partially edentulous jaws. ${ }^{4}$

In spite of the current research on the application of CBCT in the assessment of the use of restrictive guides, little literature exists involving the applications of CBCT for the production of non-restrictive guides. While restrictive guide surgery provides excellent results and great accuracy of communication between planning clinician and the implant surgeon, they prove to be quite expensive and require additional training. Restrictive guides are also not always necessary in the planning of an implant retained overdenture and could potentially prevent implant placement in situations in which some modification in site position is necessary during surgery. In these situations placement 
could still occur and provide a successful restoration with a non-restrictive guide, by providing the implant surgeon with the space needed for drill manipulation. Nonrestrictive guided surgery can provide good results with a much lower financial burden on the patient and the clinic. CBCT planning is still useful in the planning of these guides in order to plan for the bucco-lingual width of the available alveolar bone as well as the location of mandibular canal within the bone, which is not always apparent in traditional two-dimensional radiography. 


\section{CHAPTER III}

\section{MATERIALS AND METHODS}

\section{Hypothesis}

It was hypothesized that the use of radiographic markers in mandibular stents to project the clinically derived placement of implant fixtures would not correspond to the available bone based on acceptable implant placement criteria applied on Cone Beam Computed Tomography (CBCT) images.

\section{Research Design}

This investigation was designed as an observational retrospective comparison of clinically projected versus radiographically optimal implant fixture placement in totally edentulous mandibles. A retrospective radiographic audit of cone beam computed tomography (CBCT) database of patients referred for imaging to assess the status of the residual alveolar ridge (RAR) in totally edentulous mandibles prior to implant fixture placement was performed. Using implant planning software, comparisons and correlations were made between the virtual positions of projected implant fixture placement within the RAR ascertained by cylindrical radiopaque markers on surgical guides to optimal placement guidelines. A single rater (PI) performed all measurements on three separate occasions. Frequency tabulations and measurement means and standard deviations were calculated and descriptively analyzed. 


\section{Subject Selection}

The initial subject sample consisted of all patients in the available CBCT imaging database who had been referred to image the RAR in the edentulous region in either the maxilla or mandible for potential implant fixture placement. Patients had been referred either internally from within the University of Louisville School of Dentistry or externally from practitioners in private dental practice. This CBCT imaging referral service is operated as the faculty private practice by Drs. Allan G. Farman and William C. Scarfe. Both are board certified and licensed specialists in Oral and Maxillofacial Radiology in the Commonwealth of Kentucky. All CBCT images were acquired using the i-CAT ${ }^{\mathrm{TM}}$ Classic CBCT unit (Imaging Sciences International, Hatfield, PA, USA). The device was operated at $1-3 \mathrm{~mA}$ and $120 \mathrm{kV}$ using a high frequency, constant potential, fixed anode with a nominal focal spot size of $0.5 \mathrm{~mm}$. Each patient was positioned into the device supported by the constructed plastic head holder. The hard tissue chin of each patient was inserted into the chin holder and vertical and horizontal laser lights on the device used to position the head. The head was oriented such that the mid-sagittal was perpendicular to the floor and the horizontal laser reference was along an imaginary line at the intersection of the porion - orbitale (Frankfort Horizontal). Resolution was usually set at $0.4 \mathrm{~mm}$ however sometimes for the maxilla or dental impactions 0.3 or $0.25 \mathrm{~mm}$ resolutions are used. Scans could be performed at one of three volume sizes; $13.2 \mathrm{~cm}, 8 \mathrm{~cm}$ or $6 \mathrm{~cm}$ heights.

Institutional Review Board (IRB) approval was granted on March $17^{\text {th }} 2010$ (IRB \#10.0103). This study involved a retrospective audit of CBCT report records within a database (Filemaker Pro v.8, FileMaker Inc., Santa Clara, CA) held within Radiology and 
Imaging Sciences, Dept. of Surgical/Hospital Dentistry at the University of Louisville School of Dentistry, Louisville, Kentucky. Seven specific data fields were extracted from these records:

1) Date of scan. Date that the CBCT scan was obtained.

2) Patient Name. (last name, first).

3) Date of Birth. (DOB).

4) Sex. Dichotomous categorical data indicating the sex of the patient imaged in the format: $M / F$

5) Reason for referral. Categorical structured text categorizing the reason that the patient was referred for a CBCT scan. Categories included Hand Wrist, Implant $\mathrm{CBCT}$, Pathology $\mathrm{CBCT}$, Fracture $\mathrm{CBCT}$, TMJ CBCT, TMJ Tomography, Trauma CBCT, Cleft Lip/Palate, Ortho CBCT, Third Molar CBCT, Cephalometric, Sleep Apnea, Dento/Craniofacial, Impaction CBCT, Surgical follow up - plates/graft, Surgical follow up - recurrence, Surgical follow up - trauma, Consultation

6) Referring clinician name. Categorical structured text providing the name of the referring clinician prescribing the $\mathrm{CBCT}$ scan.

7) Radiologic findings. Narrative text data describing any modifications to the scan procedure and describing the imaging features of the condition. 
8) Radiologic Impression. Narrative text data summarizing the primary and incidental or secondary imaging findings.

Patient waivers were not necessary, as all personal health information was stripped from the data set collected for analysis.

The above fields were exported from a total of 2,067 radiographic reports to an spreadsheet (Excel, Microsoft Corp., Redmond, WA) representing all patients in the database from installation of the CBCT equipment in the Dept. (May 13, 2004) to a convenience date (December 31, 2009). From the data fields, two (2) additional descriptive data fields were derived:

1. Age - Numeric data identifying the age of the patient in whole years in the format: yy. This was determined by subtracting the date the scan was performed from the DOB.

2. Practitioner Type - Categorical structured text data identifying the specialty type of practitioner who referred the patient for the cone beam computed tomography. Categories included OMFS = Oral and maxillofacial surgery; GP $=$ General dental practitioner $; \mathrm{P}=$ periodontist Pros $=$ Prosthodontist $)$.

Records were first sorted by the date the scan was performed from the most recent to the earliest. The database was then sorted according to the reason for referral field.

The following inclusion and exclusion criteria were applied to the available radiographic reports (Figure $\mathrm{C} 1$ ).

1) The following search terms were applied to the reports and those including at least one of the terms independently color coded; edentulous, radiographic 
marker(s), radiographic stent, surgical guide, implant. This resulted in 884 datasets that were not suitable for inclusion into the study.

2) The remaining 1,183 datasets were then reviewed to determine the location of the potential implant fixture placement site. All records in which the potential implant fixture site(s) were located in the maxilla only were excluded (327 cases).

3) The remaining 856 reports were then reviewed to determine the dentition status of the remaining mandibular scans. The datasets of all partially edentulous patients were excluded from consideration (637 datasets).

4) The remaining 219 datasets were viewed individually using proprietary acquisition software (Xorancat, Imaging Sciences International, Hatfield, PA, USA) to determine if a surgical guide was used and if so what type of guide that was in place (outline-type guides (a), spherical "BB" type markers (b), wire over-lay type markers (c), or cylindrical markers (d) (Figure C2). All patient datasets in which markers other than cylindrical markers were excluded (142 datasets). The resultant dataset $(n=77)$ comprised mandibular totally edentulous patients in which CBCT scans were performed using surgical guides with cylindrical markers. 


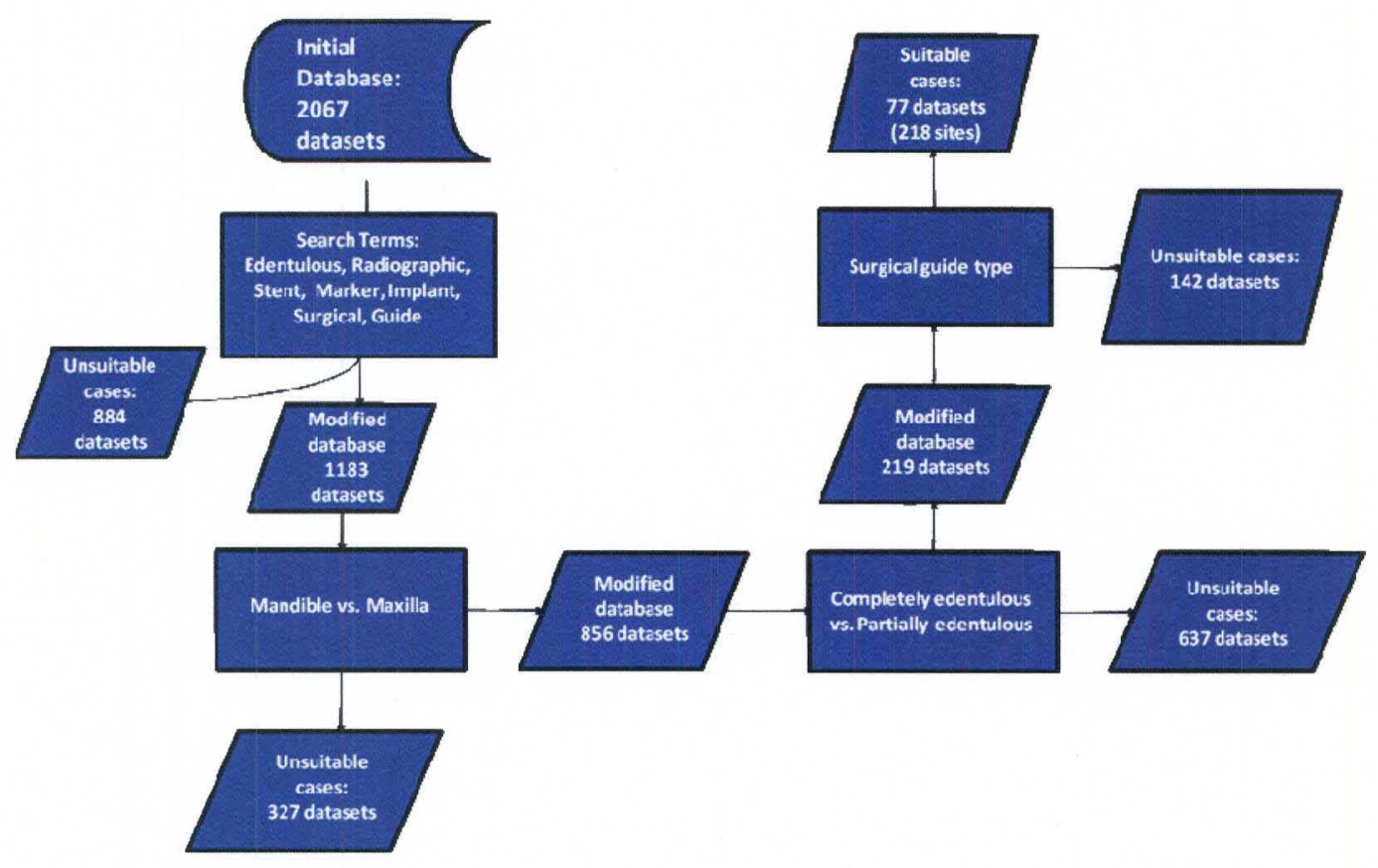

Figure C1. Subject Record Selection Process Flowchart.

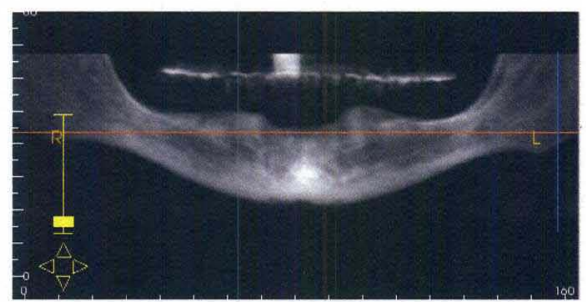

a

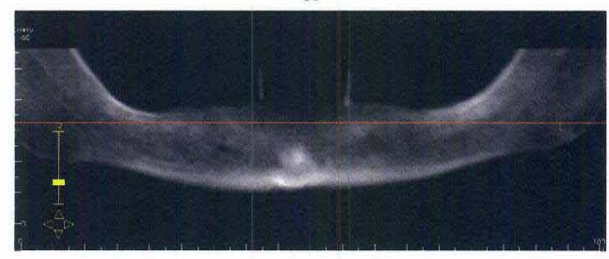

c.

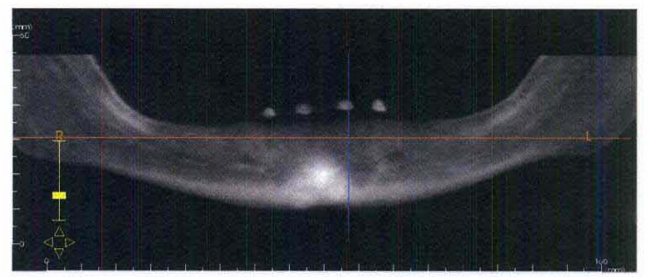

b.

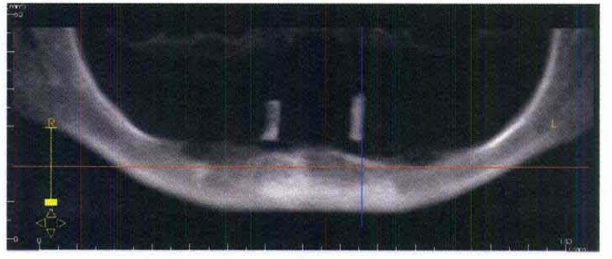

d.

Figure C2. Representative reconstructed $20 \mathrm{~mm}$ panoramic oblique planar images demonstrating varying imaging appearances of marker systems used in surgical guides. (a) Tooth outline marker, (b) Spherical (BB) type markers, (c) Wire onlay type markers, d) Cylindrical markers 
Those CBCT datasets in which patients were determined to fulfill all inclusion/exclusion criteria for the study were then exported as a DICOM files. These files were then imported into a proprietary implant analysis and planning software (EasyGuide V1.3; Aspen Dental, Burlington, MA). Upon import the DICOM data is converted into a proprietary format (EasyGuide, *.ezg) and subsequently saved as such. At this stage all fields were stripped of patient identifiers and files anonymized and saved as coded files (PtA, PtB, PtC,...PtAA, PtAB, PtAC....etc).

\section{Data Collection}

For each patient subject, the following demographics were recorded.

1) Proposed Implant Fixture Information.

a. Number of cylindrical radiographic markers present in each patient.

From this the frequency distribution, mean number of implants and median \# of sites were calculated.

b. Position of each cylindrical radiographic marker. All patients prior to CBCT imaging had been examined and a tentative treatment plan developed by a licensed practitioner with the aid of clinical examination, study models and panoramic extraoral imaging. It was assumed that surgical guides were constructed such that the position of each radiographic marker corresponded to a proposed implant fixture placement specific for the individual.

2) Patient Demographics 
a. Patient Mean Age - Average age (in years) of the sample population.

b. Patient Median Age - Median age (in years) of the sample population.

c. Standard Deviation of Patient Age - Standard deviation of patient age across sample population.

d. Sex Ratio - Number of males to number of females of the sample population.

For each patient subject the CBCT data was retrieved using the EasyGuide software and each proposed site was analyzed qualitatively and quantitatively using two display options. The "Implant Planning" display screen allowed placement and orientation of virtual implant fixture animations (Figure C3, a). The "Analysis" display allowed measurements to be obtained both on individual implant fixture animations and between multiple implant fixture animations. (Figure C3, b) Using the implant planning display, animated, theoretical standardized generic parallel implant fixtures $(10 \mathrm{~mm} \times$ $3.5 \mathrm{~mm}$ ) were then placed in the residual alveolar ridge along a line extrapolating the trajectory of the cylindrical radiographic marker on the surgical guide at each site. A second animated, theoretical implant fixture was then placed at the same site and reoriented to fulfill optimal implant placement guidelines while maintaining as little deviation from the original projected implant fixture as possible. Specifically the guidelines outlined for optimal implant placement for this study include:

1) At least $1 \mathrm{~mm}$ of alveolar bone surrounding the implant fixture on both the buccal and lingual side.

2) At least $3 \mathrm{~mm}$ of alveolar bone separating the implant fixture from adjacent implant fixtures and tooth root structures. 
3) At least $2 \mathrm{~mm}$ of alveolar bone separating the implant fixture from the mandibular canal.

4) At least $5 \mathrm{~mm}$ of alveolar bone distal to the fixture and mesial to the mental foramen in cases in which an anterior loop is observed.

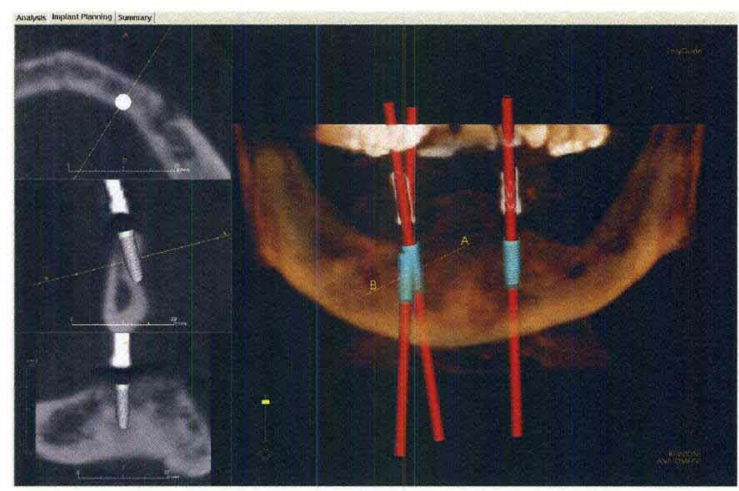

a

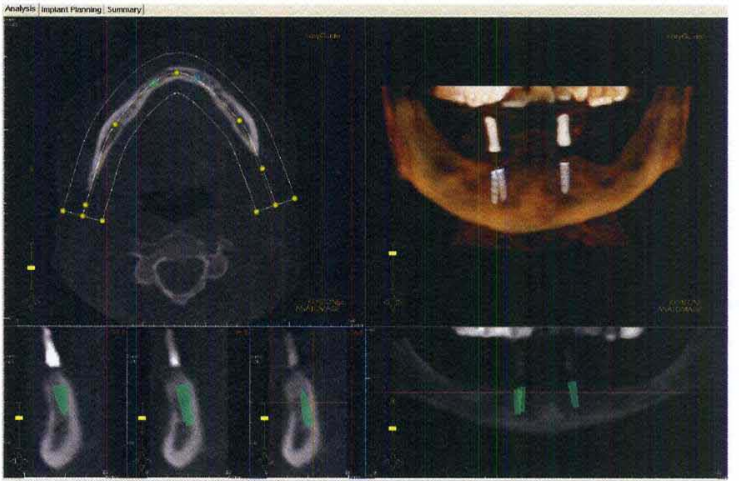

$\mathrm{b}$

Figure C3: Implant planning and Analysis functions in EasyGuide. (a) The implant planning function displays 3D reconstruction and CT slices and is used manipulate individual theoretical implant fixtures in three dimensions, (b) The analysis function displays CT, 3D reconstruction, and Panoramic images.

\section{Qualitative Assessment}

By analyzing the placement of the implant based on the projected fixture site location, the following information was derived as a descriptor of common projected complications:

1) Location of projected implant. Observational parameter defining the direction of necessary translation (if possible) of the projected implant in order to fulfill optimal implant fixture placement criteria. (Acceptable, buccal to radiographically optimal, lingual to radiographically optimal, or placement 
at projected site unlikely due to complicating factors (i.e. lack of alveolar bone or location of the mandibular canal). (Figure $\mathrm{C} 4$ )

2) Status of projected implant. Observational parameter defining projected complications with regard to projected implant fixture placement. (i.e. dehiscence, fenestration, out of bone, complications with mandibular canal, and complications due to amount of available alveolar bone) (Figure C5)

3) Status of guides containing unsuitable projections. Observational parameter defining the overall number of radiographic markers available in a surgical guide containing one or more unsuitable projections. Results are recorded according to the number of remaining acceptable sites.

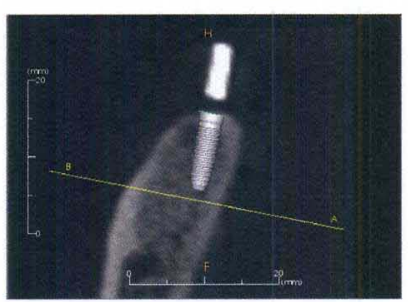

a

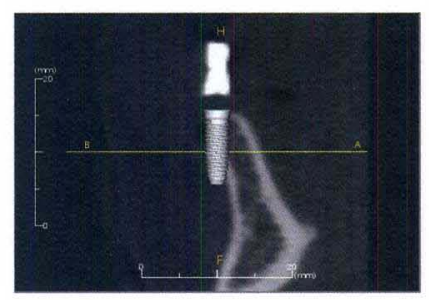

b

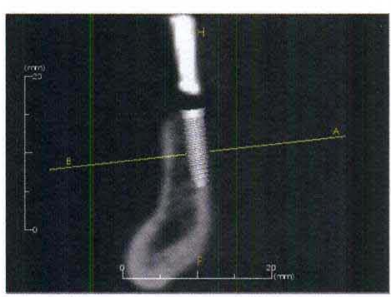

C

Figure C4: Acceptable (a), buccal (b), and lingual (c) projections with respect to available alveolar bone.

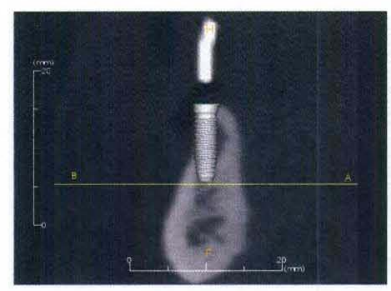

a

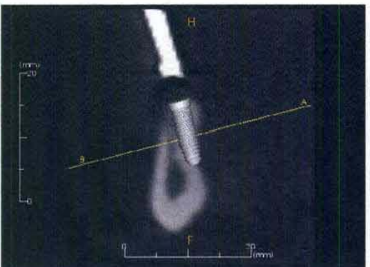

B

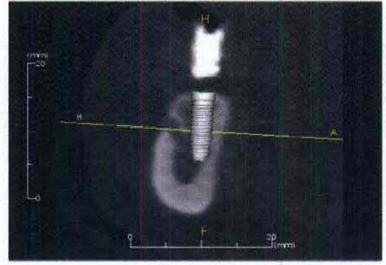

c

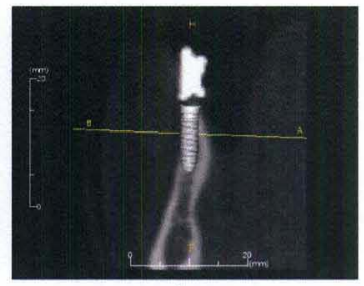

d

Figure C5: Projected placement complications: Buccal dehiscence (a), Lingual fenestration (b), Mandibular canal (c), Lack of alveolar width (d) 


\section{Quantitative Assessment}

By comparing the placement of the virtual implant based on the projected fixture site location to the optimal virtual placement the following were calculated:

1) Platform linear difference. Buccal-lingual distance between projected and radiographically optimal implant fixtures measured at the platform. (Figure C6a)

2) Apical linear difference. Buccal-lingual distance between projected and radiographically optimal implant fixtures measured at the apex. (Figure C6a)

3) Alveoloplasty. Coronal-apical distance between projected and radiographically optimal implant fixtures measured at the platform. (Figure C6b)

4) Angular deviation. Difference in angulation between optimal and projected fixture placement. (Figure C7)

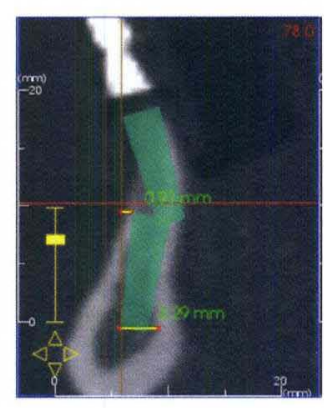

A

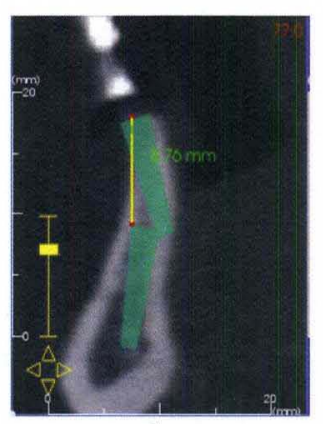

b

Figure C6: Linear platform and apical difference (a) and alveoloplasty (b) between projected and optimal theoretical implant fixtures. 


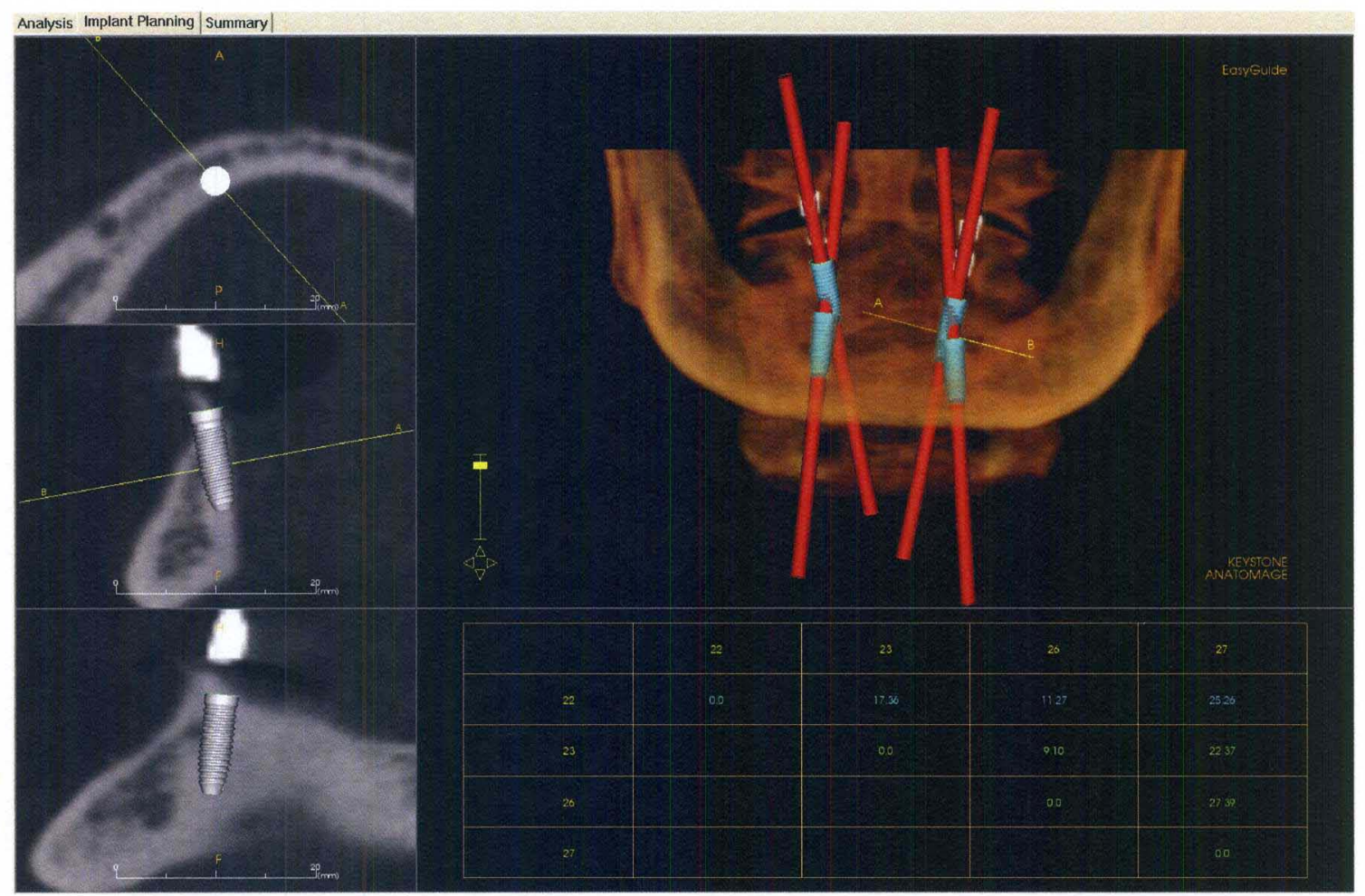

Figure C7: Angular deviation between projected implants as calculated by EasyGuide.

The placement of projected and optimal virtual implant fixture placement was repeated 3 times and measurements recorded. The average of the three measurements was then used as an estimator of the true deviation of the projected implant fixture from optimal.

The following secondary parameters were analyzed in cases in which they were applicable:

1) Available restorative space. Measure of space available for the planned restoration. This was performed in cases in which there were projections located in the posterior mandible. Due to the construction and design of 
the surgical guides this is the only area where this parameter is measurable. This measurement was performed by measuring the coronalapical distance of the top of the surgical guide to the crest of the alveolar bone. (Figure C8a)

2) Projected Placement Trajectory (PPT) vs. Residual Bone Trajectory. Angular difference of the PPT from the projection of the RBT. Due to the variable shape of residual mandibular bone, this measure was taken where possible. (Figure C8b)

3)

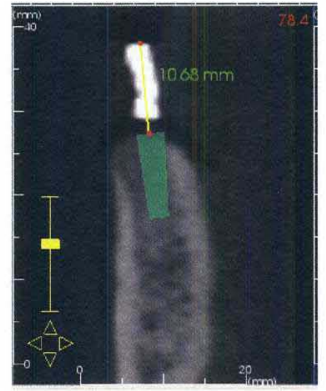

A

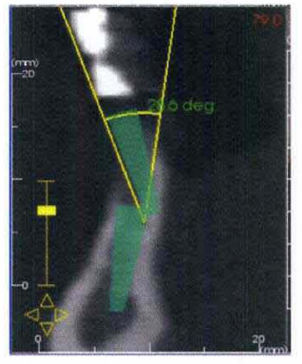

b

Figure C8: Measurement of available restorative space (a) and measurement of PPt vs.

RBT.

\section{Statistical Analysis}

The average of the three separate recordings between projected and optimal implant fixture placement, was calculated. The mean squared difference was also calculated as a measure of reliability for each parameter.

1) Mean Number of Site Projections - Average number of projected implant fixtures for each individual in the study. 
2) Median Number of Site Projections - Median number of projected implant fixtures for each individual in the study.

3) Placement Status - Ratio of acceptable implant fixtures to fixtures that can be placed with manipulation and to fixtures that cannot be placed at the projected location. (Acceptable: Possible with manipulation: Not Possible)

4) Complication Status - Ratio of the projected status over all implants with respect to projected complications. (Buccal/Lingual Dehiscence, Buccal/Lingual Fenestration, Buccally/Lingually Out of Bone)

The following statistics were calculated for the primary measurement parameters to determine to overall average deviation of the projected implant fixtures from their nearest optimal positioning:

1) Average Lateral Platform Deviation - The average lateral distance at the platform of any given projected implant fixture from optimal implant placement criteria. Measurement from the platform of the projected implant to the platform of the radiographically ideal implant (Buccal-Lingual). Three separate measurements taken and averaged for each projection.

2) Average Lateral Apex Deviation - The average lateral distance at the apex of any given projected implant fixture from optimal implant placement criteria. Measured from the apex of the projected implant to the apex of the radiographically ideal implant (Buccal-Lingual). Three separate measurements taken and averaged for each projection.

3) Average Angular Deviation - The average angular difference of any given projected implant fixture from optimal implant placement criteria. 
Calculations are made automatically by the software. Three separate measurements taken and averaged for each projection.

4) Average Alveoloplasty - Average necessary bone reduction required for the fulfillment of ideal implant placement criteria for any given projected implant fixture. Measured from the platform of the projected implant to the platform of the radiographically ideal implant (Coronal-Apical). Three separate measurements taken and averaged for each projection.

The following statistics were measured for the secondary measurement parameters:

1) Average Angular Deviation of Projected Implant Trajectory from Residual Bone Trajectory - Average angular deviation of any given projected implant fixture trajectory from the measured trajectory of the mandibular bone. Three separate measurements taken and averaged for each projection.

2) Average Available Restorative Space in the Posterior Mandible - Average measure of space available for the restoration. Measured from the crest of the mandibular bone to the top of the surgical guide projection. Three separate measurements taken and averaged for each projection. 


\section{CHAPTER IV}

\section{RESULTS}

The descriptive statistics for this study are provided in three sections. The first section describes the status of the surgical guides used to identify the clinically derived projected orientation and location of the implant fixture. The second section describes the demographics of the subject sample. Finally qualitative and quantitative statistical measures are provided regarding the location and status of the projected individual implant fixture projections compared to their ideal position determined radiographically.

\section{Surgical Guide Status}

The frequency distribution of surgical guide markers in the subject sample $(n=77)$ is shown in Figure D1. The results show that the majority of surgical guides $(n=56,73 \%)$ contained two radiographic markers (implant fixture projections) per guide. Surgical guides providing four markers accounted for the next most frequent surgical guide $(n=15$, $19.5 \%$ ) The remaining $7.5 \%$ of the scans were composed of surgical guides containing five $(n=2,3 \%), 6(n=1,1 \%), 8(n=1,1 \%), 10(n=1,1 \%)$, or $12(n=1,1 \%)$ radiographic markers. 


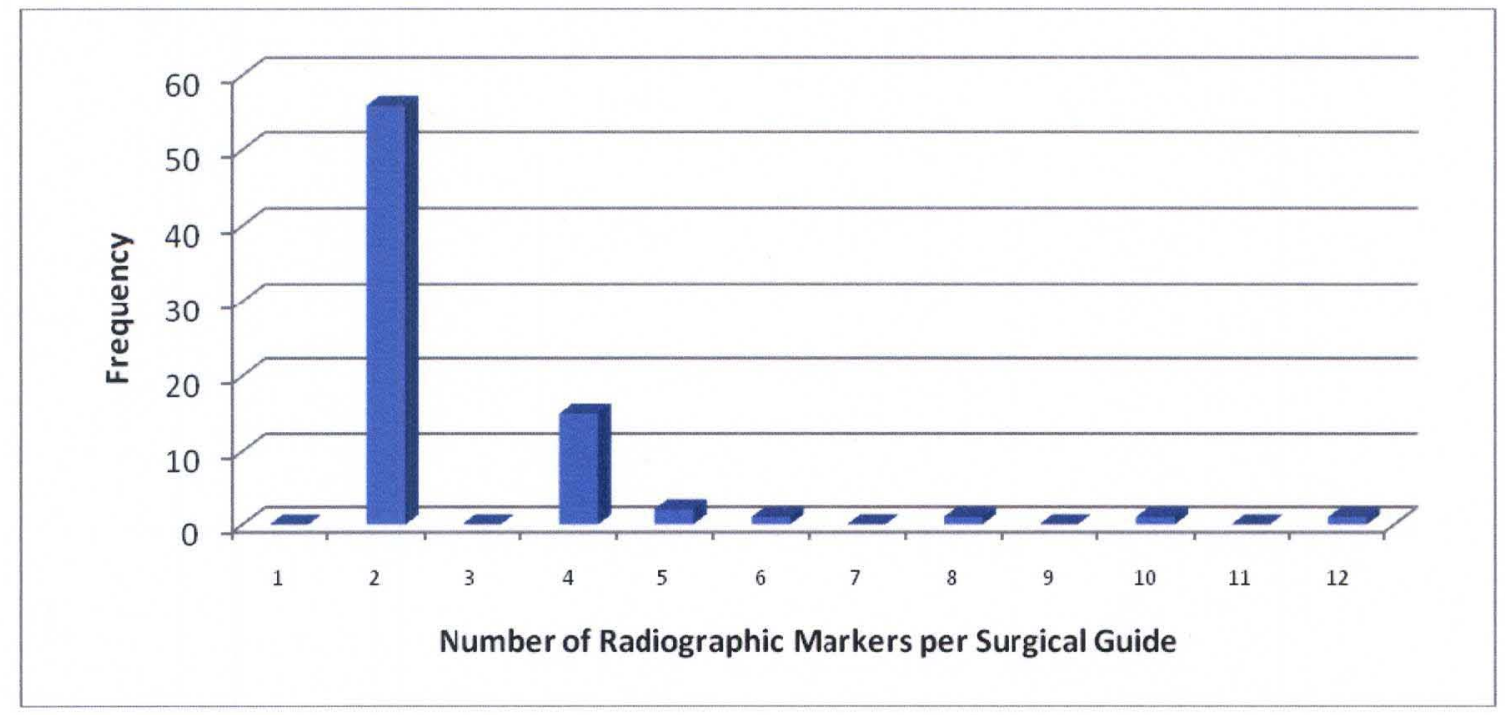

Figure D1: Frequency distribution of surgical guides by the number of radiographic markers per guide.

Figure D2 shows the frequency of individual radiographic markers $(n=219)$ grouped according to the nearest estimated tooth site. Potential sites were numbered according to the following system: $1=$ Central Incisor, $2=$ Lateral Incisor, $3=$ Canine, 4 $=$ First Pre-Molar, 5 = Second Pre-Molar, 6 = First Molar, and 7 = Second Molar. We found that the greatest number of radiographic markers were placed at site \#3, corresponding to the canine region $(\mathrm{n}=154 ; 70.3 \%)$. The next most frequent marker was placed at the first pre-molar region (site \#4) $(n=33,15.1 \%)$ with the remaining of projections ( $\mathrm{n}=32,14.6 \%)$ evenly distributed between the remaining regions. 


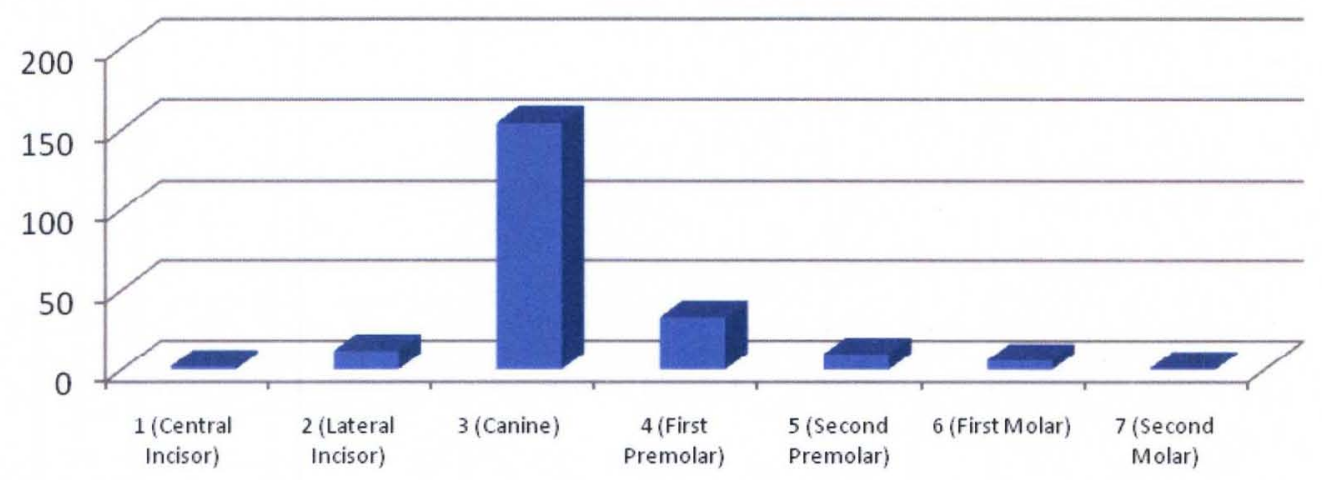

Figure D2: Frequency distribution of radiographic markers according to projected location.

\section{Subject Sample Demographics}

The mean ( \pm standard deviation) age of the subject sample was $60.7 \pm 10.4$ years (Range: $33-88$ years) with a median age of 63 years. The female to male sex ratio of the subject sample $(n=77)$ was $45: 32$.

\section{Analysis of Projected Fixture Placement}

\section{Relative to Alveolar Bone Status}

Figure D3 shows the assessment of the projected fixture placement as determined by the radiographic markers on the surgical guide in relation to the topography of the available residual alveolar ridge. This was achieved by analyzing the placement of theoretical, animated implant fixtures long the axis as indicated by the radiographic markers such that the fixture platform would be level at the crest of the residual alveolar ridge. The status of the projection was classified according to three possible situations 1) Acceptable, 2) Placement possible with fixture/bone manipulation, or 3) Placement not 
likely due to the status of the residual alveolar ridge. Fourteen (14) (6\%) projections resulted in implant placement that was acceptable without any modification. One hundred and seventy ( $\mathrm{n}=170 ; 78 \%)$ of the projections located at sites in which optimal implant placement criteria could be fulfilled with re-orientation of the trajectory and/or modification of the alveolar bone. However $34(16 \%)$ of the implant fixture projections were found to be difficult to place in the projected locations due to complicating factors such as lack of alveolar bone width, lack of alveolar bone height, or the location of the mandibular canal within the alveolar bone.

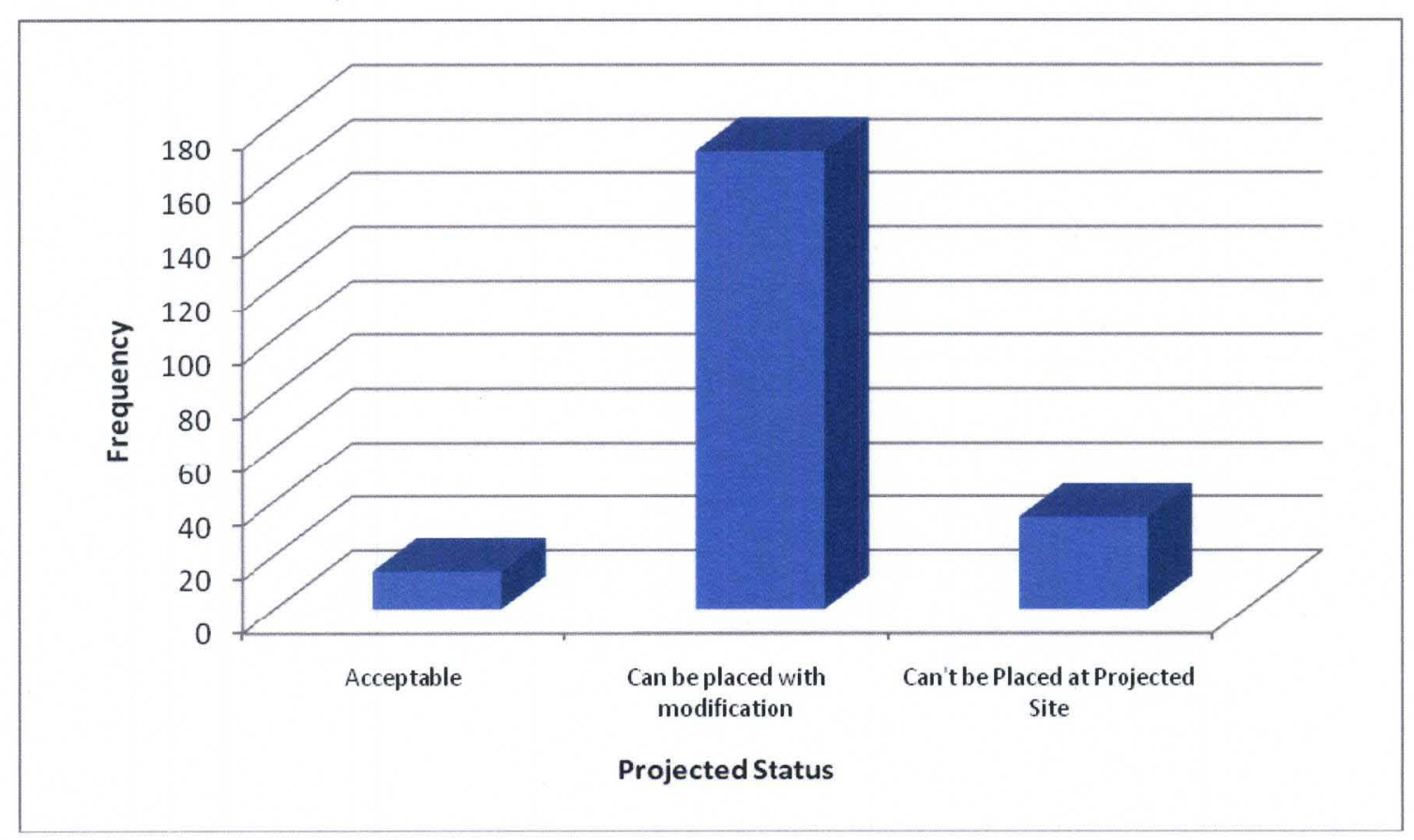

Figure D3: Primary projected implant fixture placement status.

Figure D4 shows the frequency of unsuitable sites for implant fixture placement versus the number of radiographic markers in each surgical guide. The surgical guide with the highest number of unsuitable radiographic projections occurred in the guides containing only two radiographic markers $(n=3)$. There were also surgical guides 
containing four markers $(n=1)$ and five markers $(n=1)$ that had no radiographic projections in suitable locations for implant fixture placement. There were also surgical guides containing two radiographic markers $(n=1)$ and four radiographic markers $(n=1)$ that only had one projected site that proved suitable for implant fixture placement. The remaining surgical guides $(n=5)$ were found to have at least two or more suitable implant placement sites.

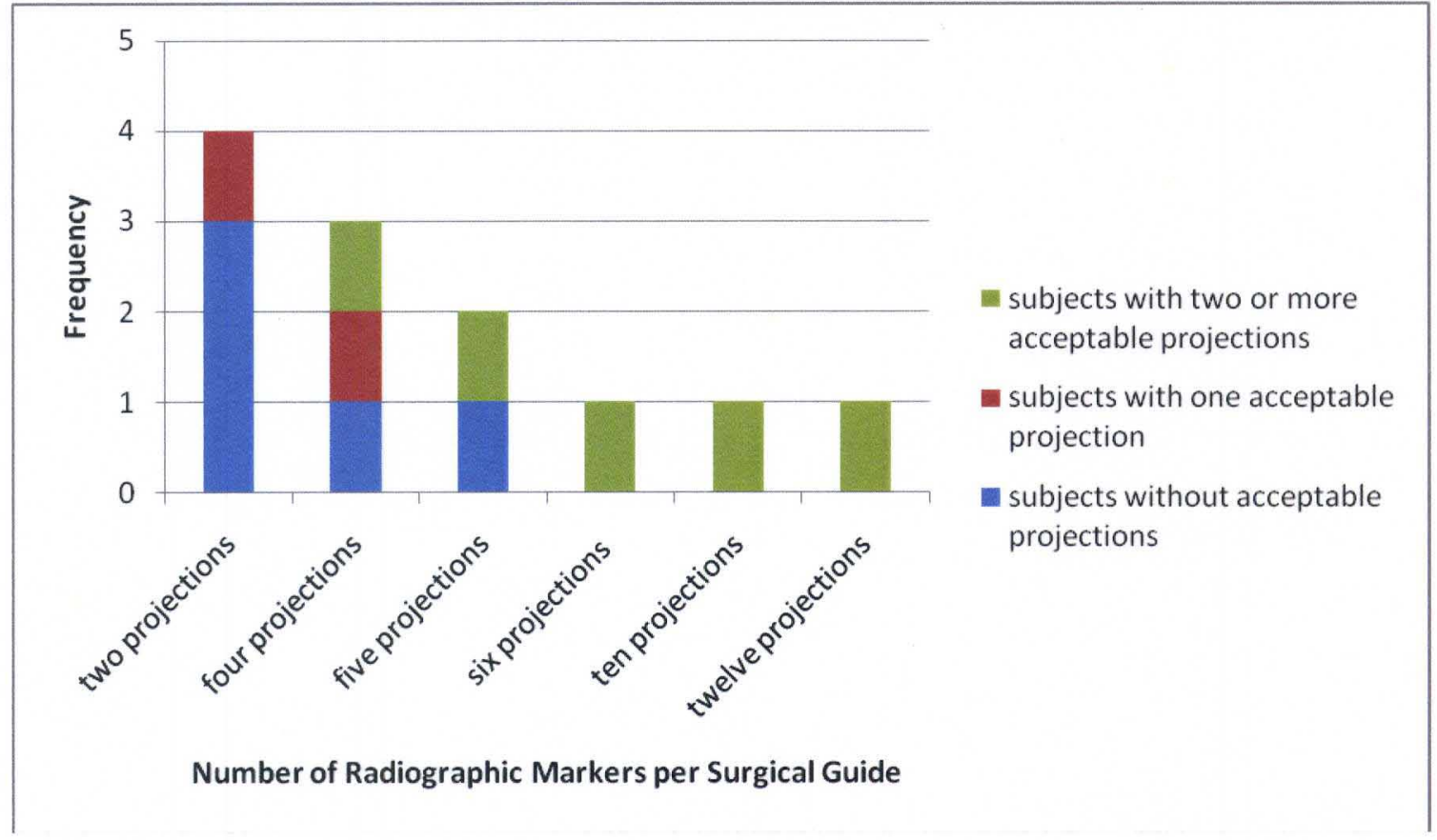

Figure D4: Acceptable radiographic projections in surgical guides containing at least one unsuitable projection with respect to the number of radiographic markers per surgical guide.

\section{Relative to Radiographically Optimal Position}

Figure D5 shows the status of the projected implant fixture with regard to buccolingual translation necessary to fulfill optimal implant fixture placement criteria. $42 \%$ 
$(\mathrm{n}=91)$ of the projected sites required lingual translation before placement, whereas $10 \%$ $(\mathrm{n}=21)$ of projections required buccal translation. The remaining projections either had an acceptable projected trajectory $(\mathrm{n}=72,33 \%)$ (e.g. alveoloplasty may still be necessary) or they were located at a site in which placement was unlikely due to complicating factors (e.g. location of the mandibular canal) $(n=34,15 \%)$.

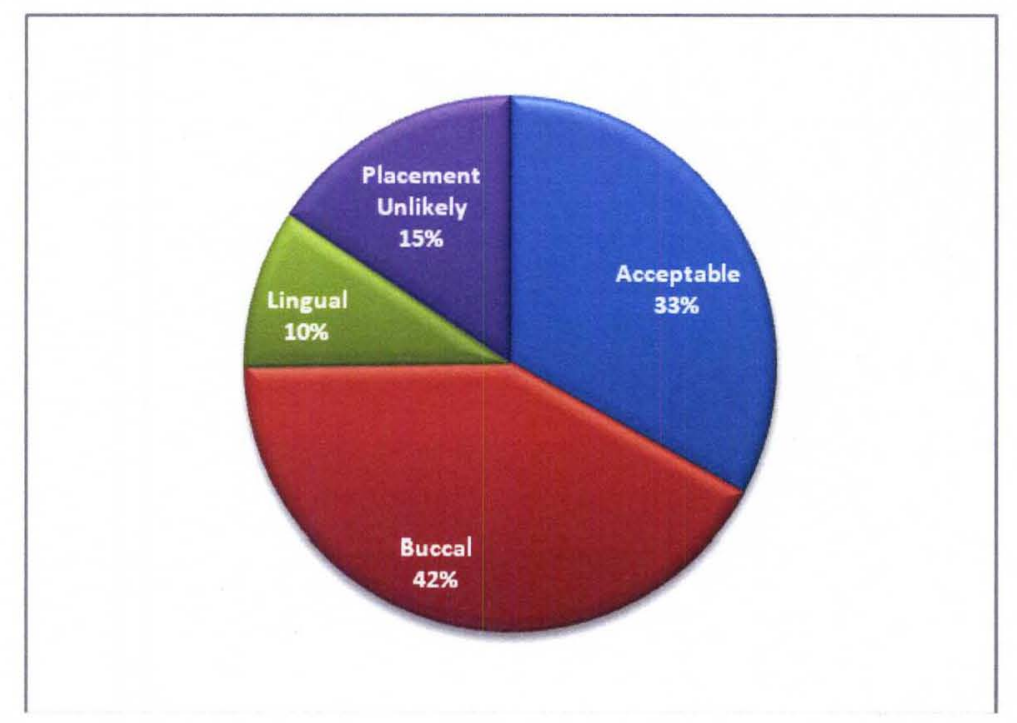

Figure D5: Projected implant fixture status with regard to optimal placement parameters.

Figure D6 shows shows the status of the projected implant fixture with regard to bucco-lingual translation in which optimal placement criteria could be fulfilled. $50 \%$ $(n=91)$ of the projections were buccal to the nearest optimal location, $39 \%(n=72)$ were acceptable, and $11 \%(\mathrm{n}=21)$ were projected to be lingual to optimal placement. 


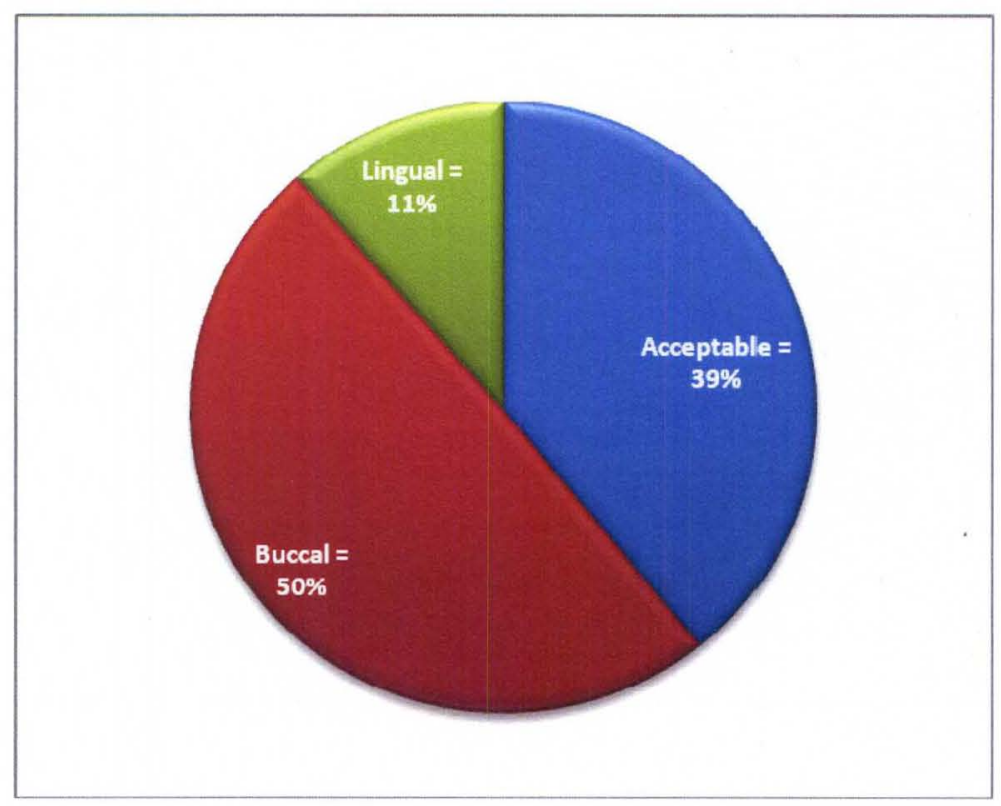

Figure D6: Projected implant fixture status with regard to optimal placement parameters for acceptable sites only.

Figure D7 shows the potential complications of implant placement at the projected sites with respect to the projected implant location without correction. The most common observed complication was a buccal dehiscence $(n=87 ; 40 \%)$. Forty four $(\mathrm{n}=44,20 \%)$ of the projections were found to have no observable complications, however, some re-orientation of the projected trajectory and/or residual alveolar ridge was necessary in order for the implant fixture to fulfill optimal implant placement criteria. Seventeen $(n=17,8 \%)$ of the projections were found to be completely outside of the residual alveolar bone on the buccal aspect. Eighteen $(n=18,8 \%)$ projections resulted in a lingual dehiscence alone. Fourteen $(n=14,6 \%)$ of the projections were found to fulfill optimal placement parameters. Fourteen $(n=14,6 \%)$ projections resulted in a lingual fenestration alone. Four $(n=4,2 \%)$ of the projections were found to be completely out of the residual alveolar bone on the lingual aspect. Five $(n=5,2 \%)$ of the 
projections were found to result in both buccal and lingual dehiscence. Five $(n=5,2 \%)$ of the projections were found to result in a buccal dehiscence and a lingual fenestration. Three $(n=3,1 \%)$ of the projections were found to result in a buccal fenestration. One $(\mathrm{n}=1,<1 \%)$ projection was found to result in both a buccal dehiscence and a buccal fenestration. One $(\mathrm{n}=1,<1 \%)$ projection resulted in a lingual dehiscence and a lingual fenestration. One $(\mathrm{n}=1,<1 \%)$ projection was found to result in a lingual dehiscence and a buccal fenestration.

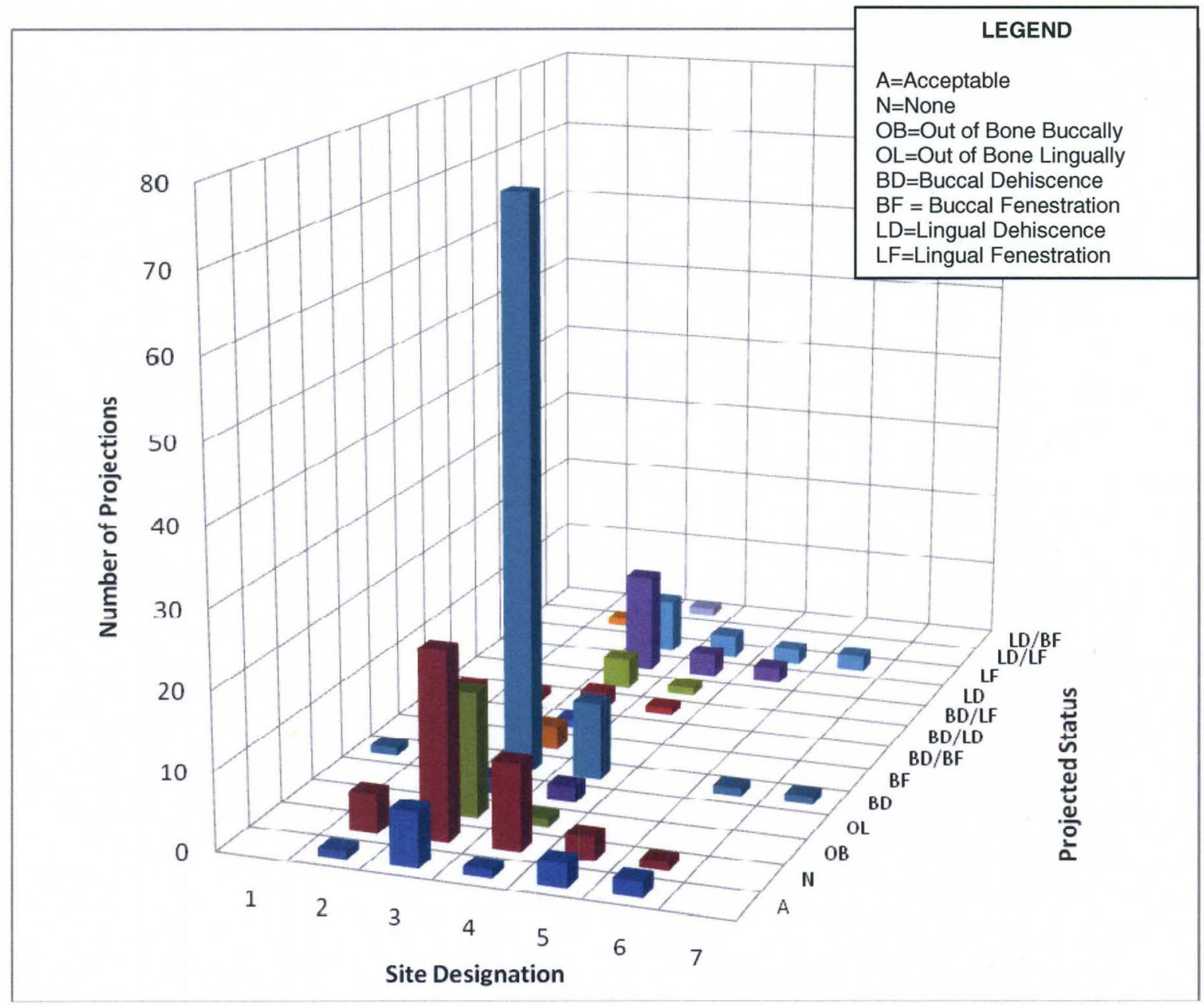

Figure D7: Potential complications with projected implant trajectories, sorted according to projected site. 
Figure D8 shows the modifications in implant fixture trajectory necessary to fulfill optimal implant fixture placement guidelines. The most common modification needed was a combination of alveolar bone reduction and lateral translation $(\mathrm{n}=80,37 \%)$ followed by alveoloplasty only $(n=58,26 \%)$. Twenty three $(n=23,10 \%)$ of the projected sites were considered unacceptable due to the proximity of the mandibular canal to the projected implant fixture site. Twenty $(n=20,9 \%)$ of the projected sites were found to need a combination of alveoloplasty, angulation, and lateral translation in order to fulfill the criteria for ideal implant fixture placement. The remaining implant fixture projections were comprised of projections that were acceptable $(6 \%, n=14)$, needed lateral translation only $(5 \%, \mathrm{n}=11)$, were unsuitable due to a lack of alveolar bone width $(4 \%, \mathrm{n}=8)$, were unsuitable due to lack of alveolar bone height $(1 \%, \mathrm{n}=3)$, or that needed only lateral translation and angulation $(0.5 \%, \mathrm{n}=1)$.

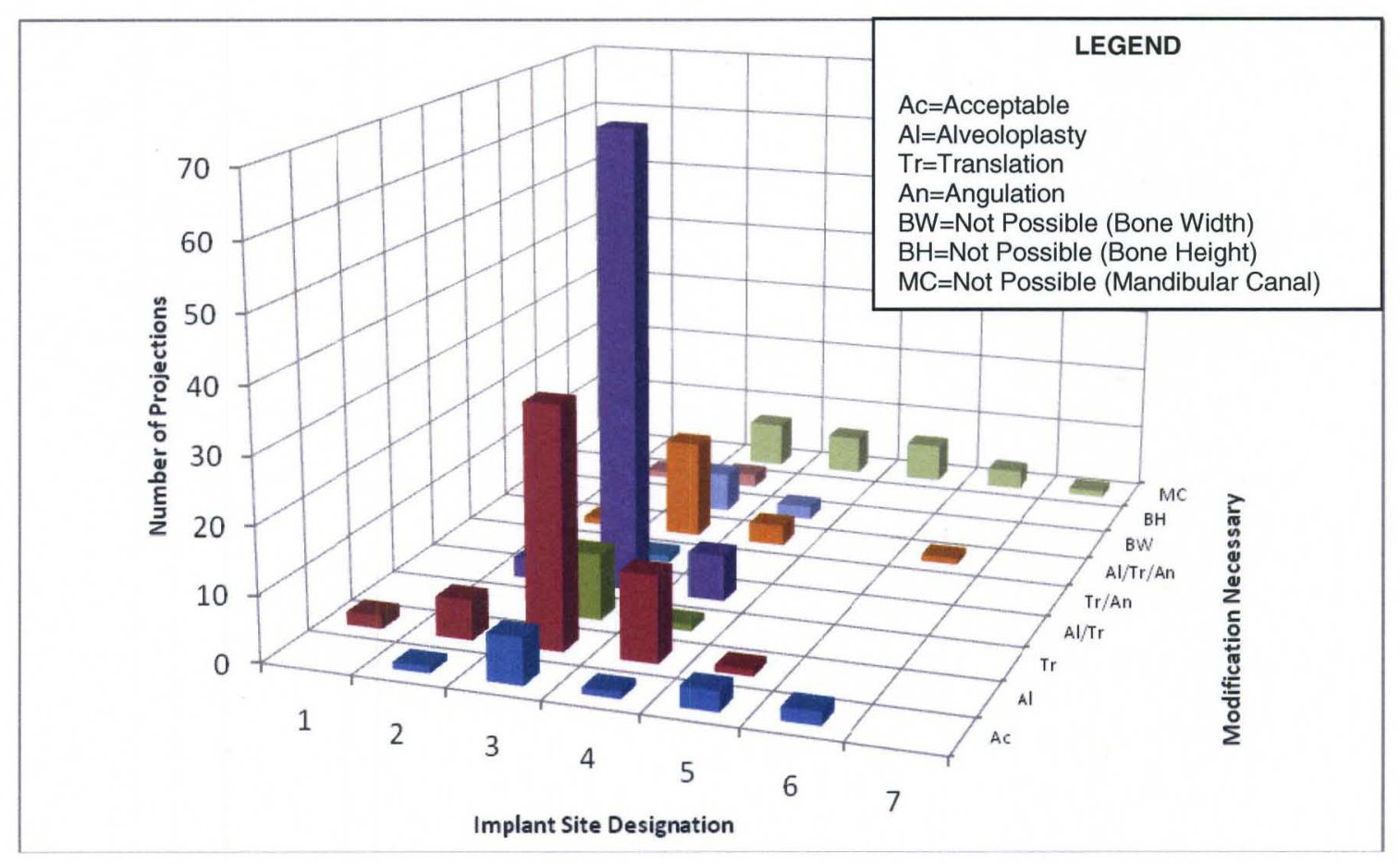


Figure D8: Graphical representation of necessary modifications to implant fixture trajectory based on site.

Figure D9 shows the average measured difference between projected and radiographically optimal implant fixture projections as well as the values for the secondary measures of average available restorative space and average projected implant trajectory vs. residual alveolar ridge trajectory. The mean alveoloplasty necessary for each implant to fulfill ideal implant placement criteria was $3.87 \pm 2.51 \mathrm{~mm}$. The average angular deviation between projected implant fixtures and radiographically ideal implant fixtures was $14.03 \pm 5.54^{0}$. The average platform translation necessary was $2.05 \pm$ $1.29 \mathrm{~mm}$. The average apical translation needed to fulfill optimal implant placement criteria was $2.29 \pm 1.49 \mathrm{~mm}$. The average angular difference between the residual alveolar ridge and the projected implant fixture trajectory was found to be $16.32 \pm 7.26^{\circ}$. The average restorative space available in the posterior mandible was found to be $11.62 \pm$ $2.60 \mathrm{~mm}$. 


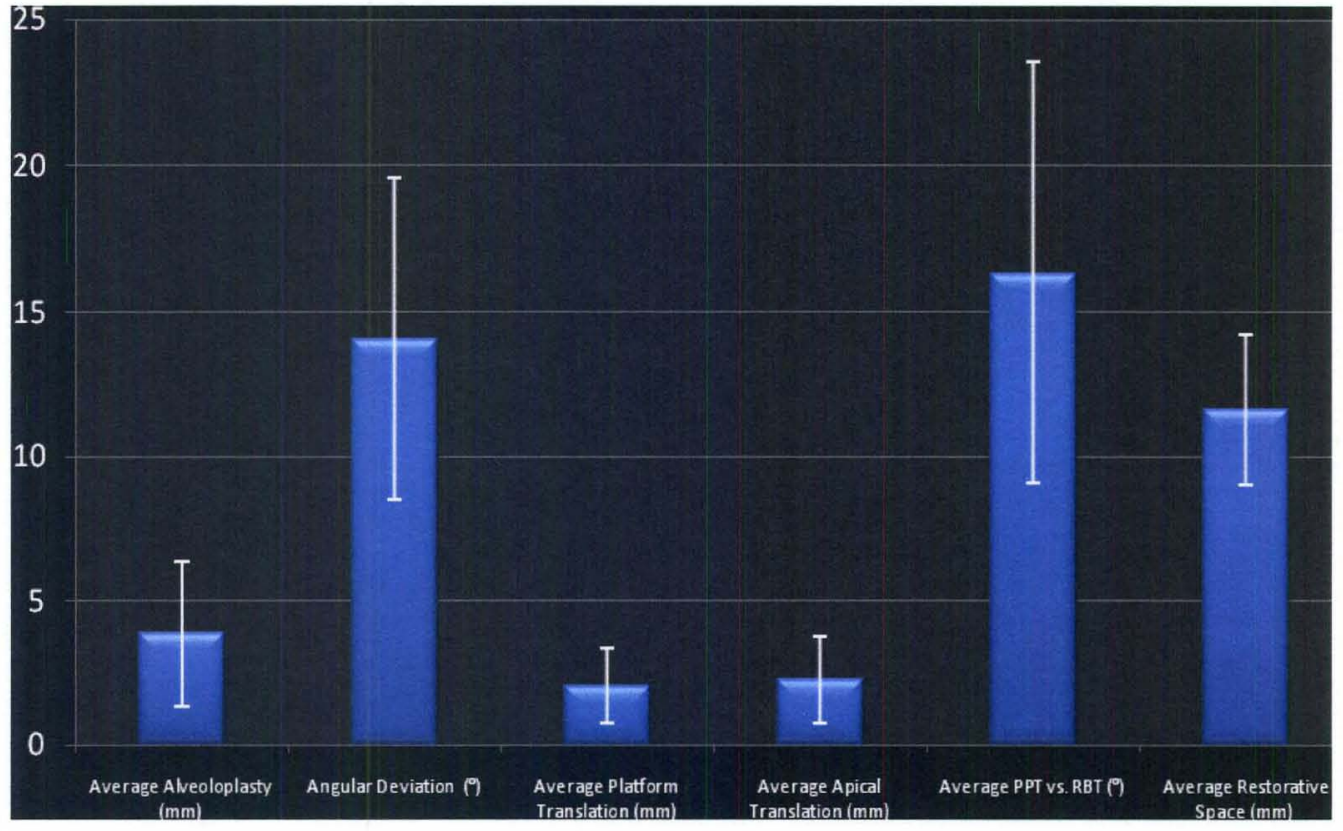

Figure D9: Quantitative analysis between projected and radiographically ideal implant fixtures. 


\section{CHAPTER V}

\section{DISCUSSION}

\section{Purpose and Motivation}

The purpose of this study was to compare the projected trajectory of implant fixture placement as determined clinically and identified by radiographic markers on surgical guides to optimal virtual implant fixture placement on rendered $\mathrm{CBCT}$ datasets in a subject sample treatment planned for a implant retained mandibular overdenture. The iCAT ${ }^{\mathrm{TM}}$ cone beam computed tomographic unit was installed in Radiology and Imaging Science at the University of Louisville School of Dentistry on May 13, 2004. During the first 6 years and 7 months (through December 31, 2010), 2,067 patient CBCT scans were performed. Seventy seven (77) patients whose CBCT datasets fulfilled the inclusion/exclusion criteria for this study acted as the subject sample for this study. Patients were referred for CBCT imaging from both within the university and from external private practices.

Demographic data was collated regarding the subject sample and type of surgical guide placed. In addition, qualitative and quantitative data was also developed by comparing clinically derived projected implant fixture to optimal virtual placement.

The demographics of the subject population indicate that the majority of patients who were potential candidates for two-implant retained mandibular overdenture were 
older (median; 63 years) - however the age range varied from 33 to 88 years. The subject sample contained slightly more females than males.

Most of surgical guides ( $73 \%$ ) contained only two radiographic markers. This was somewhat expected as the purpose of the study was to analyze edentulous mandibles that were being imaged for the purpose of planning two implant overdentures. The remaining $27 \%$ of the surgical guides contained four or more projections. For the purpose of this study these surgical guides were preferable in that if one or more projections proved unsuitable for implant fixture placement, as long as at least two of the remaining projections were suitable, they could still be analyzed and planning for the two implant overdenture could proceed. However, if one of the projected sites for a surgical guide containing two markers was unsuitable, the guide was not suitable for the purpose of the study. While in clinical practice the guide could be used, it was not possible to extrapolate the marker placement mesio-distally as a parameter for this study.

Of the 34 projected locations that were considered unsuitable for implant placement, six were found to be in patients that had only two projections in which both were unsuitable. For these patients, implant fixture placement for retention of the overdenture would not be considered possible. Eight of the projections that were considered not possible were in patients where the surgical guide comprised only four projections and all locations were considered unlikely for implant placement. For these patients, implant fixture placement for retention of the overdenture would be considered not possible. The remaining 20 projections were located in surgical guides containing four or more projections, meaning that even though there were unsuitable projections within the guide, at least two projections could be considered suitable and placement of 
implant fixtures for that subject could proceed. This demonstrates the advantage of providing multiple markers in each surgical guide to enable greater specificity through multiple reference points.

We also found that most $(70.3 \%)$ projections were located at the canine region of the mandible. This was expected, as this area generally provides the greatest amount of alveolar bone and is the most common location for the placement of implant fixtures in two-implant retained mandibular overdentures. This site was followed by projections in the first premolar region (15.1\%). This was also somewhat expected as it is near the canine region and provides many of the same benefits as the canine region for the support of an implant fixture. The remaining projected sites were distributed evenly throughout the remaining sites. The distribution of the projected implant fixture sites followed the distribution of the number of radiographic markers per surgical guide. The molar region is an undesirable location for implant fixture placement in the subject sample because of the increased potential for complications involving the mandibular canal within the alveolar bone of the posterior mandible. The anterior mandible is also undesirable compared with the canine region because of the potential of complications due to inadequate alveolar bone width as well as difficulty in the support of the restoration for implant fixtures placed in the incisor regions.

Analysis of the location of the projected implant fixture indicates that only $6 \%$ of projected radiographic marker trajectories results in optimal placement as defined by criteria. In addition, $16 \%$ of the projected implant fixture sites were unsuitable for placement based on either the amount of residual alveolar bone (either buccal-lingually or coronal-apically) or based on the location of the mandibular canal within the alveolar 
bone. The remaining $78 \%$ of the implant fixture projections could be placed at their projected locations, however, some manipulation of the trajectory would be necessary to optimize fixture placement. It is also important to note that of the $16 \%$ of the projected sites that were considered unsuitable for placement, $25 \%$ of those projections were obtained from surgical guides containing only two radiographic markers. This implies that, based on the projections, the fixture placement for the implant retained overdenture wouldn't be possible. However, $42 \%$ of these surgical guides contained more than two projections resulting in two or more acceptable projections which could be utilized for implant fixture placement for an implant retained overdenture. The remaining 33\% either had more than two sites, but all were unacceptable (17\%), or only had one acceptable projection (17\%).

Based on analysis of projected to optimal fixture placement, $15 \%$ of sites were unsuitable for implant placement. Approximately 33\% of the projected trajectories resulted in an acceptable trajectory within residual alveolar bone; however, alveoloplasty could still be a required modification even if then implant fixture trajectory results in fulfillment of the optimal implant placement criteria. $10 \%$ of the projected trajectories resulted in an implant fixture that would be lingual to the optimal implant placement criteria, and therefore would require a lateral translation in the buccal direction in order to be considered acceptable for the purposes of the study. The remaining $42 \%$ of the projections resulted in implant fixtures that were lingual to optimal criteria and would therefore require a lateral translation in the lingual direction to fulfill the optimal placement criteria. Excluding the projections at the unacceptable locations, we found only $11 \%$ of projections were lingual to optimal criteria, requiring buccal translation for 
placement. $39 \%$ of the projections were found to be acceptable, implying that the trajectory would result in fulfillment of optimal placement criteria, without regard to necessary bone reduction. The remaining $50 \%$ of projections were found to need lateral translation in the lingual direction in order to fulfill optimal placement parameters. These results are preferable for the purpose of an implant retained overdenture, as lingual translation is most often preferred for the support of the restoration.

Analyzing potential complications due to insertion of implant fixtures using clinically projected implant fixture placement alone found that the most common complication would be buccal dehiscence (42\%). This was expected due to the large number of implants analyzed as requiring lingual translation in order to fulfill optimal placement criteria. The frequency of occurrence was especially noticeable at sites \#3 and \#4 (canine and pre-molar regions) due to the large number of projections at that site. The second most common occurrence would have been lack of alveolar bone surrounding the projected fixture ( $20 \%$ of the projected implant fixtures). These were especially noticeable at sites $\# 2, \# 3$, and \#4. There were also a large number of radiographic marker trajectories that resulted in implant fixtures that were more than $50 \%$ extruded buccally $(8 \%)$. This was most prevalent at site \#3. $6 \%$ of the projected implant fixtures resulted in acceptable placement without any modification to the projected trajectory or the alveolar bone. This occurred most commonly at sites \#3, \#5, and \#6. The instance of lingual dehiscence $(8 \%)$ and lingual fenestration $(6 \%)$ were also seen, particularly at site \#3. The remaining $10 \%$ of projected implant fixture placement complications were composed of combinations of the earlier defined complications. 
A combination of alveoloplasty and lateral translation $(37 \%)$ was the most common manipulation necessary for those discrepancies in projected implant fixture placement to fulfill the optimal implant fixture criteria. This corresponds closely to the frequency of dehiscence in the analysis of projected complications. The second most frequent modification necessary between projected and radiographically optimal implant fixtures was alveoloplasty alone (26\%), which suggests an acceptable trajectory by the referring clinician, but perhaps an unaccounted for structural defect or abnormal bone shape. This was followed by a combination of angulation, alveoloplasty, and lateral translation (9\%) which corresponded with projected complications of dehiscence/fenestration and fenestration alone. The remaining projections were either acceptable, requiring no modification at all (6\%), or placement at the projected location was unlikely due to the location of the mandibular canal (11\%), lack of alveolar bone width $(4 \%)$, or lack of alveolar bone height $(1 \%)$.

\section{Limitations of this study}

One limitation of this study is that subject inclusion was restricted to those patients referred for CBCT imaging to only one facility. While this resulted in a relatively small subject sample size $(\mathrm{n}=77)$ providing a relatively small number of implant fixture projections $(\mathrm{n}=218)$, this also limited the numbers of operators involved in planning the location and projection of proposed implant fixtures, thereby reducing the clinical variability in surgical guide construction due to greater consistency of the referring clinicians. 
The study also lacked a clearly defined "gold standard" defining the final position of the projected implant fixtures. While all of the parameters outlined and utilized to analyze the implant placement provide an ideal situation for implant fixture placement, there are still factors, both known (dental hygiene, parafunctional habits, etc) and unknown, which affect the overall success rate of dental implants.

The retrospective nature of the study is also limiting to some degree. There was no way of determining what final treatment plan was utilized in each case, or if implants actually were placed. A prospective study could have provided more information for each individual subject.

The estimation of implant fixture size as being constant across all study subjects also limits the estimation of the potential treatment plan. While a generic $3.5 \times 10 \mathrm{~mm}$ implant provides a good estimate of an implant fixture that would generally be used, there are still other options available (e.g. mini-implants) that could be placed in situations where the generic implant fixture would prove unacceptable.

\section{Future Areas of Research}

Future research could include a similar analysis of the restorative tooth-outline type surgical guide rather than the cylindrical marker type radiographic markers. This would require greater estimation of the placement of the implant fixture since the projection is based on the restoration rather than the implant fixture itself.

Research could also expand into the area of projections based on single tooth implants rather than overdentures. This would, however, require more factors to be taken into considerations, such as measurement parameters regarding location of surrounding 
tooth structures and the end restoration orientation with respect to the surrounding structures.

It would also be interesting, in future studies, to include an analysis of long-term individual implant fixture success based on the planning processes with respect to guidetype and placement locations within the alveolar ridge. This would require much greater time and financial investment. 


\section{CHAPTER VI}

\section{CONCLUSION}

In this study, we retrospectively analyzed the clinically derived projected implant fixture position based on cylindrical radiographic markers on surgical guides for totally edentulous patients on CBCT images and compared this to optimal placement criteria. . The trajectories of the radiographic markers were descriptively analyzed with reference to their resultant projections location within residual alveolar bone. The projections were also analyzed with respect to projected complications, deviation from fulfilling radiographically ideal conditions, manipulations necessary in order to fulfill those conditions, and the number of projections per surgical guide as well as the location of those projections.

The results of this study suggest that the residual alveolar bone is not always available in the location that the referring clinician believes it to be. This study also suggests that a greater number of radiographic markers can provide better reference and overall planning for implant fixture placement in cases in which some of the projected sites prove to be unsuitable. Finally, the use of non-restrictive surgical guides allow the implant surgeon to make modifications in site position and still place implants based on the final restoration. 


\section{REFERENCES}

1. Ersoy AE, Turkyilmaz I, Ozan O, McGlumphy EA. (2008) Reliability of implant placement with stereolithographic surgical guides generated from computed tomography: Clinical data from 94 implants. J Periodontol 79:1339-1345.

2. Nickenig H, Eitner S (2007) "Reliability of implant placement after virtual planning of implant positions using cone beam CT data and surgical (guide) templates." Journal of Cranio-Maxillofacial Surgery 35:207-211.

3. Horwitz J, Zuabi O, Machtei EE. (2009) Accuracy of a computerized tomography-guided template-assisted implant placement system: an in vitro study." Clin. Oral Impl. Res; xx:000-000.

4. Van Assche N, van Steenberghe D, Guerrero ME, Hirsch E, Schutyser F, Quirynen M, Jacobs R. (2007) "Accuracy of implant placement based on presurgical planning of three-dimensional cone-beam images: a pilot study." J Clin Periodontol 34:816-821.

5. Carter L, Farman AG, Geist J, Scarfe WC, Angelopoulos C, Nair MK, Hildebolt CF, Tyndall D, Shrout M. (2008) American Academy of Oral and Maxillofacial Radiology executive opinion statement on performing and interpreting diagnostic cone beam computed tomography. Surg Oral Oral Med Oral Pathol Oral Radiol Endod. 106(4):561-562

6. Jalbout Z, Tabourian G ed. (2008) Glossary of Implant Dentistry II, International Congress of Oral Implantologists, Upper Montclair, NJ. p. 84

7. Almog DM, Torrado E, Meitner SW. (2001) Fabrication of imaging and surgical guides for dental implants. J Prosthet Dent. 85:504-508.

8. Burns DR, Crabtree DG, Bell DH. (1988) Template for positioning and angulation of intraosseous implants. J Prosthet Dent. 60:479-83.

9. Higginbottom FL, Wilson TG. (1996) Three-dimensional templates for placement of root-form dental implants: a technical note. Int J Oral Maxillofac Implants 11:787-93.

10. Meitner SW, Tallents RH. (2004) Surgical templates for prosthetically guided implant placement. J Prosthet Dent 92:569-74 
11. Greenstein G, Cavallaro J. (2007) The relationship between biological concepts and fabrication of surgical guides for dental implant placement. Compend Contin Edu Dent 28(4): 196-204

12. Tyndall DA, Brooks SL. (2000) Selection criteria for dental implant site imaging: a position paper of the American Academy of Oral and Maxillofacial radiology. Oral Surg Oral Med Oral Pathol Oral Radiol Endod. 89:630

13. DelBalso AM, Greiner FG, Licata M. (1994) Role of diagnostic imaging in the evaluation of the dental implant patient. Radiographics;14:699-719.

14. Adell R, Lekholm U, Rockler B, Brånemark P-I. (1981) A 15-year study of osseointegrated implants in the treatment of the edentulous jaw. Int J Oral Surg 10:387-416.

15. Greenstein G, Tarnow D (2006) The Mental Foramen and Nerve: Clinical and Anatomical Factors Related to Dental Implant Placement: A Literature Review. Journal of Periodontology 77:1933-1943.

16. Misch CE, Goodacre CJ, Finley JM, Misch CM, Marinbach M, Dabrowsky T, English CE, Kois JC, Cronin RJ. (2004) Crown-Height Space Guidelines for Implant Dentistry. Consensus Conference Panel Report. Oral Implantology.

17. Sadowsky SJ. (2001) Mandibular Implant Retained Overrdentures: A Literature review. J Prosthet Dent. 86:468-73.

18. http://www.branemark.com/History.html

19. Binon, P (2000) Implants and Components: Entering the New Millinium. Int J of Oral \& Maxillofacial Implants. 15:76-95. 


\section{CURRICULUM VITAE}

NAME: $\quad$ William Shane Vaughn

ADDRESS: 1525 S. $3^{\text {rd }}$ St.

Apartment \#7

Louisville, KY 40208

DOB: $\quad$ Hopkinsville, Kentucky - September 14, 1984

EDUCATION AND TRAINING:

B.S. Chemistry / Biology

Kentucky Wesleyan College

2002-2006

AWARDS:

PROFESSIONAL SOCIETIES:

PUBLICATIONS:

NATIONAL MEETING PRESENTATIONS:

REFERRED JOURNALS:

BOOKS AND SYMPOSIA:

INVITED PRESENTATIONS: 